\title{
FREE-BOOLEAN INDEPENDENCE WITH AMALGAMATION
}

\author{
WEIHUA LIU AND PING ZHONG
}

\begin{abstract}
In this paper, we develop the notion of free-Boolean independence in an amalgamation setting. We construct free-Boolean cumulants and show that the vanishing of mixed free-Boolean cumulants is equivalent to our free-Boolean independence with amalgamation. We also provide a characterization of free-Boolean independence by conditions in terms of mixed moments. In addition, we study free-Boolean independence over a $C^{*}$-algebra and prove a positivity property.
\end{abstract}

\section{INTRODUCTION}

Free probability theory is a probability theory that studies noncommutative random variables with highest noncommutativity. This theory, due to Voiculescu, is based on the notion of free independence which is an analogue of the classical independence. In [18, Voiculescu generalized his notion of free independence to free independence with amalgamation over an arbitrary algebra in details. To be specific, moments of random variables are no longer scalar numbers but elements from a given algebra. On the other aspect, Voiculescu started to study pairs of random variables simultaneously thereby generalized the notion of free independence to a notion of bi-free independence [19]. Further more, the notion of bi-free independence with amalgamation, defined by Voiculescu [19], was fully developed in [2]. there are exactly two unital universal independence relations, namely Voiculescu's free independence relation, the classical independence relation [15]. It was mentioned that we would obtain more independence relations by decreasing the number of axioms for universal products [8]. For instance, people introduced Boolean independence [17], monotone independence [10, conditionally independence [1] in various contexts. Their operator-valued generalization were studied as well [9, 12, 13]. Recently, their corresponding independence relations for pairs of random variables, analog of Voiculescu's bi-free theory, were introduced and studied [4, 3, 6]. Furthermore, the conditionally bi-free independence with amalgamation is studied in [5].

In [8], the first-named author introduced a notion of mixed independence relations for pairs of random variables, where random variables in different faces exhibit different kinds of noncommutative independence. In particular, the combinatorics of free-Boolean independence relation was fully developed. In this paper, we generalize the notion of free-Boolean independence to an amalgamation setting. Relevant combinatorial tools are extended to study this new independence. Beyond the corresponding combinatorial results, we address the positivity of free-Boolean independence with amalgamation. Therefore, it is possible to study the relation in topological probability spaces but not only algebraic probability spaces. For instance, we can study our 
free-Boolean independence with amalgamation over a $C^{*}$-algebra, which is a suitable framework to address some probabilistic questions. We plan to study probabilistic results such as operatorvalued infinitely divisible laws in a forthcoming paper.

The paper is organized as follows: Besides this introduction, in Section 2, we give the definition of free-Boolean independence with amalgamation over an algebra. In Section 3, we review some relevant combinatorial tools. In Section 4, we demonstrate that free-Boolean independence can be characterized by the property of the vanishing of mixed free-Boolean cumulants. In Section 5 , we prove an operator-valued version of free-Boolean central limit law. In Section 6, we provide an equivalent characterization of free-Boolean independence by certain moments-conditions. In Section 7 , we study the positivity property for free-Boolean independence relation.

\section{Preliminaries and Notations}

In this section, we give the motivation and the definition for free-Boolean independence relation with amalgamation over an algebra.

Definition 2.1. A $B$ - $B$-bimodule with a specified projection is a triple $(\mathcal{X}, \dot{\mathcal{X}}, p)$, where $\mathcal{X}$ is a direct sum of $B$ - $B$-bimodules $\mathcal{X}=B \oplus \dot{\mathcal{X}}$, and $p: \mathcal{X} \rightarrow B$ is the projection

$$
p(b \oplus \eta)=b .
$$

Denote by $\mathcal{L}(\mathcal{X})$ the algebra of linear operators with respect to the $B$ - $B$-bimodule structure. The expectation from $\mathcal{L}(\mathcal{X})$ onto $B$ is the linear map $\mathbb{E}_{\mathcal{L}(\mathcal{X})}: \mathcal{L}(\mathcal{X}) \rightarrow B$ defined by

$$
\mathbb{E}_{\mathcal{L}(\mathcal{X})}(a)=p\left(a\left(1_{B} \oplus 0\right)\right) .
$$

We now recall the definition of the reduced free product of $B$ - $B$-bimodules with specified projections [16, 18]. Let $\left\{\left(\mathcal{X}_{i}, \dot{\mathcal{X}}_{i}, p_{i}\right)\right\}_{i \in \mathcal{I}}$ be a family of $B$ - $B$-bimodules with specified projections. The reduce free product of $\left\{\left(\mathcal{X}_{k}, \dot{\mathcal{X}}_{i}, p_{i}\right)\right\}_{i \in \mathcal{I}}$ with amalgamation over $B$ is defined to be the $B$ - $B$ bimodule with a specified projection $(\mathcal{X}, \dot{\mathcal{X}}, p)$, where $\mathcal{X}=B \oplus \dot{\mathcal{X}}$ and ${ }_{\mathcal{X}}^{\circ}$ is the $B$ - $B$-bimodule defined by

$$
\dot{\mathcal{X}}=\bigoplus_{n \geq 1} \bigoplus_{i_{1} \neq i_{2} \neq \cdots \neq i_{n}}{\stackrel{\circ}{i_{1}}} \otimes_{B} \cdots \otimes_{B}{\stackrel{\circ}{\mathcal{X}_{n}}}
$$

For each $i \in \mathcal{I}$, we denote by

$$
\mathcal{X}(i)=B \oplus \bigoplus_{n \geq 1} \bigoplus_{\substack{i_{1} \neq i_{2} \neq \cdots \neq i_{n} \\ i_{1} \neq i}} \dot{\mathcal{X}}_{i_{1}} \otimes_{B} \cdots \otimes_{B} \dot{\mathcal{X}}_{i_{n}}
$$

and let $V_{i}$ be the natural isomorphism of bimodules

$$
V_{i}: \mathcal{X} \rightarrow \mathcal{X}_{i} \otimes_{B} \mathcal{X}(i)
$$

For each $i \in \mathcal{I}, \lambda_{i}: \mathcal{L}\left(\mathcal{X}_{i}\right) \rightarrow \mathcal{L}(\mathcal{X})$ is a unital homomorphism defined by

$$
\lambda_{i}(a)=V_{i}^{-1}(a \otimes I) V_{i}
$$


and $\beta_{i}: \mathcal{L}\left(\mathcal{X}_{i}\right) \rightarrow \mathcal{L}(\mathcal{X})$ is a linear map defined by

$$
\beta_{i}(a)=P_{i} \lambda_{i}(a) P_{i}
$$

where $P_{i}: \mathcal{X} \rightarrow B \oplus \dot{\mathcal{X}}_{i}$ is the natural projection onto $B \oplus \stackrel{\circ}{\mathcal{X}}_{i}$ and vanishes on the other direct summands.

Proposition 2.2. For any $a \in \mathcal{L}\left(X_{i}\right)$, we have $P_{i} \lambda_{i}(a)=\lambda_{i}(a) P_{i}$.

Proof. Notice that the reduced free product $\mathcal{X}$ of $B$ - $B$-bimodules with specified projections can be decomposed as

$$
\mathcal{X}=\left(B \oplus \stackrel{\circ}{\mathcal{X}}_{i}\right) \oplus \mathcal{X}_{i}^{\prime}, \quad \text { where } \quad \mathcal{X}_{i}^{\prime}=\bigoplus_{j \neq i} \dot{\mathcal{X}}_{j} \oplus \bigoplus_{n \geq 2} \bigoplus_{i_{1} \neq i_{2} \neq \cdots \neq i_{n}}{\stackrel{\circ}{i_{1}}} \otimes_{B} \cdots \otimes_{B}{\stackrel{\circ}{i_{n}}}
$$

The space $B \oplus \dot{\mathcal{X}}_{i}$ is invariant under $\lambda_{i}(a)$ for any $a \in \mathcal{L}\left(\mathcal{X}_{i}\right)$. We can check directly that the space $\mathcal{X}_{i}^{\prime}$ is also invariant under $\lambda_{i}(a)$ for any $a \in \mathcal{L}\left(\mathcal{X}_{i}\right)$ by the definition of $\lambda_{i}$. Hence the result follows.

The preceding result implies the next corollary.

Corollary 2.3. The map $\beta_{i}: \mathcal{L}\left(\mathcal{X}_{i}\right) \rightarrow \mathcal{L}(\mathcal{X})$ is a homomorphism.

Definition 2.4. A $B$-valued probability space is a pair $(\mathcal{A}, \mathbb{E})$ consisting of an algebra $\mathcal{A}$ over $B$ and an $B$ - $B$-bimodule map $\mathbb{E}: \mathcal{A} \rightarrow B$, i.e. a linear map such that

$$
\mathbb{E}\left(b_{1} a b_{2}\right)=b_{1} \mathbb{E}(a) b_{2}
$$

for all $a \in \mathcal{A}$ and $b_{1}, b_{2} \in B$.

Definition 2.5. Let $(\mathcal{A}, \mathbb{E})$ be a $B$-valued probability space. A family of $B$-faces of $\mathcal{A}$ is a family $\left\{\left(C_{i}, D_{i}\right)\right\}_{i \in \mathcal{I}}$ of (not necessarily unital) subalgebras of $\mathcal{A}$ such that $C_{i}, D_{i}$ are $B$ - $B$-bimodules for each $i \in \mathcal{I}$. The family of $B$-faces $\left\{\left(C_{i}, D_{i}\right)\right\}_{i \in \mathcal{I}}$ is said to be free-Boolean with amalgamation over $B$ if

- $\left\{C_{i} \mid i \in \mathcal{I}\right\}$ are unital algebras

- there are $B$ - $B$-bimodules with specified projections $\left\{\left(\mathcal{X}_{i}, \stackrel{\circ}{\mathcal{X}}_{i}, p_{i}\right)\right\}_{i \in \mathcal{I}}$ such that there are unital homomorphisms $\gamma_{i}: C_{i} \rightarrow \mathcal{L}\left(\mathcal{X}_{i}\right)$, (not necessarily unital) homomorphisms $\delta_{i}$ : $D_{i} \rightarrow \mathcal{L}\left(\mathcal{X}_{i}\right)$,

- Let $(\mathcal{X}, \dot{\mathcal{X}}, p)$ be the reduce free product of $\left\{\left(\mathcal{X}_{i}, \dot{\mathcal{X}}_{i}, p_{i}\right)\right\}_{i \in \mathcal{I}}$, so that the joint distribution of the family $\left\{\left(C_{i}, D_{i}\right)\right\}_{i \in \mathcal{I}}$ in $(\mathcal{A}, \mathbb{E})$ is equal to the joint distribution of the family of operators $\left\{\left(\lambda_{i}\left(\gamma_{i}\left(C_{i}\right)\right), \beta_{i}\left(\delta_{i}\left(D_{i}\right)\right)\right\}_{i \in \mathcal{I}}\right.$ in the probability space $\left(\mathcal{L}(\mathcal{X}), \mathbb{E}_{\mathcal{L}(\mathcal{X})}\right)$. That is,

$$
\mathbb{E}_{\mathcal{L}(\mathcal{X})}\left(\lambda_{i_{1}}\left(\gamma_{i_{1}}\left(c_{1}\right)\right) \beta_{i_{1}}\left(\delta_{i_{1}}\left(d_{1}\right)\right) \cdots \lambda_{i_{n}}\left(\gamma_{i_{n}}\left(c_{n}\right)\right) \beta_{i_{n}}\left(\delta_{i_{n}}\left(d_{n}\right)\right)\right)=\mathbb{E}\left(c_{1} d_{1} \cdots c_{n} d_{n}\right),
$$

where $c_{k} \in C_{i_{k}}, d_{k} \in D_{i_{k}}, 1 \leq k \leq n$. 


\section{INTERVAL-NONCROSSING PARTITIONS}

In this section, we review some combinatorial tools which will be used

to define operator-valued free-Boolean cumulants. We give a characterization of free-Boolean independence with amalgamation thereby generalizes results in [8] to the operator-valued framework. In noncommutative probability theory, non-crossing partitions are used in the combinatorics of free probability and the interval partitions are used in the combinatorics of Boolean independence. It turns out the partitions used in the combinatorics of free-Boolean independence are so-called interval-noncrossing partitions introduced in [8]. All results without proof in this section are taken from 8 .

3.1. Interval-noncrossing partitions. Throughout this section, we let $n \in \mathbb{N}, \chi:\{1, \cdots, n\} \rightarrow$ $\{\mathcal{F}, \mathcal{B}\}$ and $\epsilon:\{1, \cdots, n\} \rightarrow \mathcal{I}$, for some fixed index set $\mathcal{I}$. We will denote by $[n]$ the set $\{1, \cdots, n\}$ for $n \in \mathbb{N}$.

Definition 3.1. Let $S$ be a linearly ordered set. A partition $\pi$ of the set $S$ consists of a collection disjoint, nonempty sets $\left\{V_{1}, \cdots, V_{p}\right\}$ whose union is $S$. The sets $V_{1}, \cdots, V_{p}$ are called the blocks of $\pi$. Given $v_{1}, v_{2} \in S$, we write $v_{1} \sim v_{2}$ if the two elements $v_{1}, v_{2}$ are in the same block.

1. A partition $\pi$ is called noncrossing if there is no quadruple $\left(v_{1}, v_{2}, w_{1}, w_{2}\right)$ such that $v_{1}<w_{1}<v_{2}<w_{2}, v_{1}, v_{2} \in V_{s}$, and $w_{1}, w_{2} \in V_{t}$, where $V_{s}, V_{t}$ are two disjoint blocks of $\pi$. The set of all noncrossing partitions of $[n]$ will be denoted by $N C(n)$.

2. A block $V_{s}$ of $\pi$ is called interval if for any $v_{1}, v_{2} \in V_{s}$ and $v_{1}<w<v_{2}$, we have $w \in V_{s}$. A partition $\pi=\left\{V_{1}, \cdots, V_{p}\right\}$ is called an interval partition if all blocks $V_{s}$ are interval blocks.

3. A block $V_{s}$ of a partition $\pi$ is said to be inner if there is another block $V_{t} \in \pi$ and $v_{1}, v_{2} \in V_{t}$ such that $v_{1}<w<v_{2}$ for all $w \in V_{s}$. A block is outer if it is not inner.

4. Let $\epsilon:[n] \rightarrow \mathcal{I}$. We denote by $\operatorname{ker}(\epsilon)$ the partition whose blocks are the sets $\omega^{-1}(i), i \in \mathcal{I}$.

5. Given two partitions $\sigma$ and $\pi$, we say $\sigma \leq \pi$ if each block of $\sigma$ is contained in a block of $\pi$. This relation is called the reversed refinement order.

6. We denote by $0_{n}$ the partition of $[n]$ consists of $n$ blocks and by $1_{n}$ the partition of $[n]$ consists of exactly one block.

Definition 3.2. Let $\chi:\{1, \cdots, n\} \rightarrow\{\mathcal{F}, \mathcal{B}\}$. A partition $\pi$ of $[n]$ is said to be intervalnoncrossing with respect to $\chi$ if $\pi$ is noncrossing, and $v_{1}, v_{2}, w$ are in the same block whenever $v_{1}<w<v_{2}, v_{1} \sim v_{2}$ and $\chi(w)=\mathcal{B}$. We denote by $\operatorname{INC}(\chi)$ the set of all interval-noncrossing partitions of the set $\{1,2, \cdots, n\}$ with respect to $\chi$.

Remark 3.3. The set $I N C(\chi)$ does not depend on the value of $\chi$ at 1 and $n$. In particular, when $\chi^{-1}(\mathcal{B}) \cap[2, n-1]=\emptyset$, we have $\operatorname{INC}(\chi)=N C(n)$.

For example, let $\chi:\{1, \cdots, 7\} \rightarrow\{\mathcal{F}, \mathcal{B}\}$ such that $\chi^{-1}(\mathcal{B})=\{2,6,7\}$. Given two noncrossing $\pi_{1}=\{\{1,4\},\{2\},\{3\},\{5,7\},\{6\}\}$ and $\pi_{2}=\{\{1,2,4\},\{3\},\{5,6,7\}\}$ of the set $\{1, \cdots, 7\}$, then $\pi_{1} \notin I N C(\chi)$ and $\pi_{2} \in I N C(\chi)$. In pictures below, we use "•"to denote elements in $\chi^{-1}(\mathcal{F})$ 
and "॰"to denote elements in $\chi^{-1}(\mathcal{B})$.

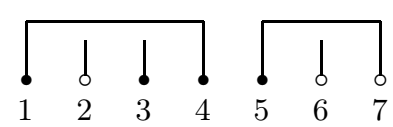

Diagram of $\pi_{1}$.

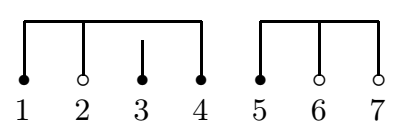

Diagram of $\pi_{2}$.

Assume now $\chi^{-1}(\mathcal{B}) \cap[2, n-1]=\left\{l_{1}<\cdots<l_{m-1}\right\}$ and set $l_{0}=0, l_{m}=n$. We denote by $\left[l_{i}, l_{i+1}\right]$ the interval $\left\{l_{i}, l_{i}+1, \cdots, l_{i+1}\right\}$. For each $i=1, \cdots, m$, we denote by $\alpha_{i}(\pi)$ the restriction of $\pi$ to the interval $\left[l_{i-1}, l_{i}\right]$. We also denote by $\alpha^{\prime}(\pi)$ the restriction of $\pi$ to the interval $\left[l_{1}, n\right]$ and $\chi^{\prime}$ the restriction of $\chi$ to the interval $\left[l_{1}, n\right]$. Note that each $\alpha_{i}(\pi)$ can be any noncrossing partition of the set $\left[l_{i-1}, l_{i}\right]$, since there is no $l_{i-1}<w<l_{i}$ such that $\chi(w)=\mathcal{B}$.

Proposition 3.4. Let $\alpha_{1}^{\prime}: I N C(\chi) \rightarrow N C\left(\left[1, l_{1}\right]\right) \times I N C\left(\chi^{\prime}\right)$ be defined by

$$
\alpha_{1}^{\prime}(\pi)=\left(\alpha_{1}(\pi), \alpha^{\prime}(\pi)\right)
$$

and $\alpha: \operatorname{INC}(\chi) \rightarrow N C\left(\left[1, l_{1}\right]\right) \times N C\left(\left[l_{1}, l_{2}\right]\right) \times \cdots \times N C\left(\left[l_{m-1}, n\right]\right)$ be defined by

$$
\alpha(\pi)=\left(\alpha_{1}(\pi), \cdots, \alpha_{m}(\pi)\right)
$$

Then $\alpha_{1}^{\prime}$ and $\alpha$ are isomorphisms of partial ordered sets. The set $I N C(\chi)$ is a lattice with respect to the reverse refinement order $\leq$ on partitions.

We provide pictures below to illustrate the preceding proposition. Let $n=10, \chi^{-1}(\mathcal{B})=$ $\{1,3,7,8,9,10\}$ and $\pi=\{\{1,3,4,7\},\{2\},\{5,6\},\{9,8\},\{10\}\}$ which is an interval-noncrossing of the set $\{1,2, \cdots, 10\}$ with respect to $\chi$ as shown in the following diagram.

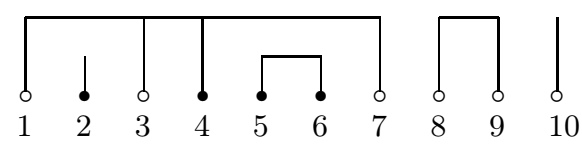

Diagram of $\pi$.

In the above diagram, $l_{0}=1, l_{1}=3, l_{2}=7, l_{3}=8, l_{4}=9, l_{5}=10$. Therefore, $\alpha_{1}(\pi)=$ $\{\{1,3\},\{2\}\}, \alpha_{2}(\pi)=\{\{3,4,7\},\{5,6\}\}, \alpha_{3}(\pi)=\{\{7\},\{8\}\}, \alpha_{4}(\pi)=\{\{8,9\}\}$ and $\alpha_{5}(\pi)=$ $\{\{9\},\{10\}\}$ are illustrated in the following diagrams: 


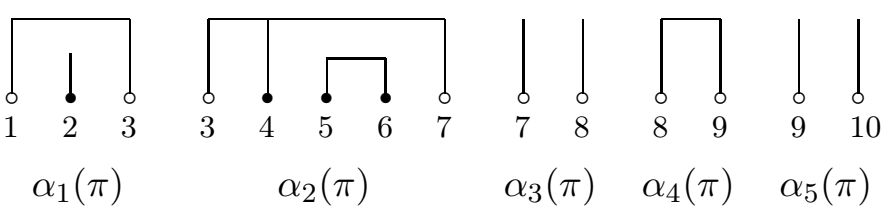

Proposition 3.5. Let $\pi=\left\{V_{1}, \cdots, V_{p}\right\} \in I N C(\chi)$ and let $\sigma \in N C(n)$ such that $\sigma \leq \pi$, i.e. each block of $\sigma$ is contained in a block of $\pi$. Then $\sigma \in I N C(\chi)$ if and only if $\left.\sigma\right|_{V_{s}} \in I N C\left(\left.\chi\right|_{V_{s}}\right)$ for all $s=1, \cdots, p$.

Proposition 3.6. Let $\pi=\left\{V_{1}, \cdots, V_{p}\right\} \in I N C(\chi)$. Denote by $\left[0_{n}, \pi\right]$ the set of all $\sigma \in I N C(\chi)$ such that $\sigma \leq \pi$. Then

$$
\left[0_{n}, \pi\right] \cong I N C\left(\left.\chi\right|_{V_{1}}\right) \times \cdots \times I N C\left(\left.\chi\right|_{V_{p}}\right) .
$$

3.2. Möbius functions on interval-noncrossing partitions. One can define the convolution for functions on the lattice following the standard procedure for partially ordered sets (see [14]). Once the map $\chi$ is fixed, the lattice structure of $I N C(\chi)$ caputred from the lattice of the product of noncrossing partitions according to the natural isomorphism described in Proposition 3.4 .

Let $\chi:\{1, \cdots, n\} \rightarrow\{\mathcal{F}, \mathcal{B}\}$. Given two complex-valued functions defined on the set $\{(\sigma, \pi) \mid \sigma, \pi \in$ $\operatorname{INC}(\chi), \sigma \leq \pi\}$. The convolution of $f$ and $g$ is given by

$$
f * g(\sigma, \pi)=\sum_{\substack{\rho \in I N C(\chi) \\ \sigma \leq \rho \leq \pi}} f(\sigma, \rho) g(\rho, \pi) .
$$

The delta function defined as follows:

$$
\delta_{I N C}(\sigma, \pi)= \begin{cases}1, & \text { if } \sigma=\pi, \\ 0, & \text { otherwise. }\end{cases}
$$

We then define the zeta function by

$$
\zeta_{I N C}(\sigma, \pi)= \begin{cases}1, & \text { if } \sigma \leq \pi, \\ 0, & \text { otherwise }\end{cases}
$$

and the Möbius function $\mu_{I N C}$ is the inverse of the zeta function in the following sense:

$$
\mu_{I N C} * \zeta_{I N C}=\zeta_{I N C} * \mu_{I N C}=\delta_{I N C}
$$

We will use the following product formula in [7, Section 6].

Proposition 3.7. Let $\chi:\{1, \cdots, n\} \rightarrow\{\mathcal{F}, \mathcal{B}\}, \pi=\left\{V_{1}, \cdots, V_{p}\right\} \in I N C(\chi)$ and $\sigma \in I N C(\chi)$ such that $\sigma \leq \pi$. Suppose that $l_{0}=1, l_{m}=n$ and $\chi^{-1}(\mathcal{B}) \cap[2, n-1]=\left\{l_{1}<\cdots<l_{m-1}\right\}$, then

$$
\begin{aligned}
\mu_{I N C(\chi)}(\sigma, \pi) & =\prod_{i=1}^{m} \mu_{I N C\left(\left.\chi\right|_{V_{i}}\right)}\left(\sigma_{i}, \pi_{i}\right) \\
& =\prod_{s=1}^{p} \mu_{I N C}\left(\left.\sigma\right|_{V_{s}}, 1_{V_{s}}\right) \\
& =\prod_{i=1}^{m} \prod_{s=1}^{p} \mu_{N C}\left(\sigma_{i} \mid \tilde{\alpha}_{i}\left(V_{s}\right), 1_{\tilde{\alpha}_{i}\left(V_{s}\right)}\right)
\end{aligned}
$$


where $\sigma_{i}=\alpha_{i}(\sigma), \pi_{i}=\alpha_{i}(\pi), 1 \leq i \leq m$ and $\tilde{\alpha}_{i}\left(V_{s}\right)$ is the restriction of $V_{s}$ to the set $\left[l_{i-1}, l_{i}\right]$.

Corollary 3.8. Let $\pi \in I N C(\chi)$ and $V \in \pi$. Denote by $\left[0_{n}, \pi\right]=\left\{\sigma \in I N C(\chi): 0_{n} \leq \sigma \leq \pi\right\}$, and $V^{\prime}=\{1, \cdots, n\} \backslash V$. Then,

$$
\left[0_{n}, \pi\right] \cong I N C\left(\left.\chi\right|_{V}\right) \times I N C\left(\chi \mid V^{\prime}\right) .
$$

In particular, we have

$$
\mu_{I N C}(\sigma, \pi)=\mu_{I N C}\left(\left.\sigma\right|_{V}, 1_{V}\right) \mu_{I N C}\left(\left.\sigma\right|_{V^{\prime}},\left.\pi\right|_{V^{\prime}}\right)
$$

for $\sigma \in I N C(\chi)$ and $\sigma \leq \pi$.

\section{VANishing CUMUlants CONDITION FOR FREE-BoOlEAN INDEPENDENCE}

In this section, we introduce the notion of operator-valued free-Boolean cumulants for pairs of random variables and give an alternative characterization of free-Boolean independence by using the free-Boolean cumulants.

4.1. Free-Boolean cumulants. Let $(\mathcal{A}, \mathbb{E})$ be a $B$-valued probability space . Let $\Phi^{(n)}$ be the $n$-B-linear map from $\underbrace{\mathcal{A} \otimes_{B} \cdots \otimes_{B} \mathcal{A}}_{\mathrm{n} \text { times }}$ to $B$ defined as

$$
\Phi^{(n)}\left(a_{1}, \cdots, a_{n}\right)=\mathbb{E}\left(a_{1} \cdots a_{n}\right) .
$$

Then, for each noncrossing partition $\pi \in N C(n)$, we can write $\pi=\pi_{1} \cup\{V\}$, where $V=$ $(l+1, l+2, \cdots, l+s)$ is an interval block of $\pi$ and $\pi_{1}=\pi \backslash\{V\}$. We define an $n$-B-linear map $\Phi_{\pi}: \underbrace{\mathcal{A} \otimes_{B} \cdots \otimes_{B} \mathcal{A}}_{\mathrm{n} \text { times }} \rightarrow B$ recursively as follows:

$$
\Phi_{\pi}\left(a_{1}, \cdots, a_{n}\right)=\Phi_{\pi_{1}}\left(a_{1}, \cdots, a_{l}, \Phi^{(s)}\left(a_{l+1}, \cdots, a_{l+s}\right) a_{l+s+1}, \cdots, a_{n}\right) .
$$

For example, let $\pi=\{\{1,5,8\},\{2,3,4\},\{6,7\}\}$ be a noncrossing partition of $\{1, \cdots, 8\}$. Then,

$$
\Phi_{\pi}\left(a_{1}, \cdots, a_{8}\right)=\Phi_{\{\{1,5,8\},\{6,7\}\}}\left(a_{1}, \mathbb{E}\left(a_{2} a_{3} a_{4}\right) a_{5}, a_{6}, a_{7}, a_{8}\right)=\mathbb{E}\left(a_{1} \mathbb{E}\left(a_{2} a_{3} a_{4}\right) a_{5} \mathbb{E}\left(a_{6} a_{7}\right) a_{8}\right) .
$$

Definition 4.1. Given any $\chi:\{1, \cdots, n\} \rightarrow\{\mathcal{F}, \mathcal{B}\}, \pi \in I N C(\chi)$ and a tuple of elements $\left(a_{1}, \cdots, a_{n}\right)$ in $(\mathcal{A}, \mathbb{E})$, the free-Boolean cumulant $\kappa_{\chi, \pi}$ is an $n$ - $B$-linear map defined as follows:

$$
\kappa_{\chi, \pi}\left(a_{1}, \cdots, a_{n}\right)=\sum_{\substack{\sigma \leq \pi \\ \sigma \in I N C(\chi)}} \mu_{I N C}(\sigma, \pi) \Phi_{\sigma}\left(a_{1}, \cdots, a_{n}\right) .
$$

We start to show that the operator-valued free-Boolean cumulants have the following multiplicative property.

Theorem 4.2. Let $\pi \in I N C(\chi)$ and $a_{1}, \cdots, a_{n}$ be noncommutative random variables in a $B$ valued probability space $(\mathcal{A}, \mathbb{E})$. Suppose that $V=(l+1, l+2, \cdots, l+s)$ is an interval block of $\pi$, then

$$
\begin{aligned}
\kappa_{\chi, \pi}\left(a_{1}, \cdots, a_{n}\right) & =\kappa_{\left.\chi\right|_{V^{\prime}},\left.\pi\right|_{V^{\prime}}}\left(a_{1}, \cdots, a_{l}, \kappa_{\left.\chi\right|_{V}, 1_{V}}\left(a_{l+1}, \cdots, a_{l+s}\right) a_{l+s+1}, \cdots, a_{n}\right) \\
& =\kappa_{\left.\chi\right|_{V^{\prime}},\left.\pi\right|_{V^{\prime}}}\left(a_{1}, \cdots,\left(a_{l} \kappa_{\left.\chi\right|_{V}, 1_{V}}\left(a_{l+1}, \cdots, a_{l+s}\right)\right), a_{l+s+1}, \cdots, a_{n}\right)
\end{aligned}
$$


where $V^{\prime}=\{1, \cdots, n\} \backslash V$.

Proof. For any $\sigma \leq \pi, \sigma \in I N C(\chi)$, one can decompose it into a union of two interval-noncrossing partitions $\sigma=\sigma_{1} \cup \sigma_{2}$, where $\sigma_{1} \leq\left.\pi\right|_{V^{\prime}}, \sigma_{1} \in I N C\left(\left.\chi\right|_{V^{\prime}}\right)$ and $\sigma_{2} \leq\left.\pi\right|_{V}=1_{V}, \sigma_{2} \in I N C\left(\left.\chi\right|_{V}\right)$. By Proposition 3.7 and Corollary 3.8, we have

$$
\begin{aligned}
& \kappa_{\chi, \pi}\left(a_{1}, \cdots, a_{n}\right)=\sum_{\substack{\sigma \leq \pi \\
\sigma \in I N C(\chi)}} \mu_{I N C}(\sigma, \pi) \Phi_{\sigma}\left(a_{1}, \cdots, a_{n}\right) \\
& =\sum_{\substack{\sigma \leq \pi \\
\sigma \in I N C(\chi)}} \mu_{I N C}(\sigma, \pi) \Phi_{\left.\sigma\right|_{V^{\prime}}}\left(a_{1}, \cdots, a_{l}, \Phi_{\left.\sigma\right|_{V}}\left(a_{l+1}, \cdots, a_{l+s}\right) a_{l+s+1}, \cdots, a_{n}\right) \\
& =\sum_{\substack{\sigma \leq \pi \\
\sigma \in I N C}} \mu_{I N C}\left(\left.\sigma\right|_{V^{\prime}},\left.\pi\right|_{V^{\prime}}\right) \mu_{I N C}\left(\left.\sigma\right|_{V}, 1_{V}\right) \Phi_{\left.\sigma\right|_{V^{\prime}}}\left(a_{1}, \cdots, a_{l}, \Phi_{\left.\sigma\right|_{V}}\left(a_{l+1}, \cdots, a_{l+s}\right) a_{l+s+1}, \cdots, a_{n}\right) \\
& =\sum_{\sigma_{1} \leq\left.\pi\right|_{V^{\prime}}} \mu_{I N C}\left(\sigma_{1},\left.\pi\right|_{V^{\prime}}\right) \mu_{I N C}\left(\sigma_{2}, 1_{V}\right) \Phi_{\left.\sigma\right|_{V^{\prime}}}\left(a_{1}, \cdots, a_{l}, \Phi_{\left.\sigma\right|_{V}}\left(a_{l+1}, \cdots, a_{l+s}\right) a_{l+s+1}, \cdots, a_{n}\right) \\
& \sigma_{1} \in I N C\left(\left.\chi\right|_{V^{\prime}}\right) \\
& =\sum_{\substack{\sigma_{1} \leq\left.\pi\right|_{V^{\prime}} \\
\sigma_{1} \in I N C\left(\left.\chi\right|_{V^{\prime}}\right)}} \mu_{I N C}\left(\sigma_{1},\left.\pi\right|_{V^{\prime}}\right) \Phi_{\sigma_{V^{\prime}}}\left(a_{1}, \cdots, a_{l},\right. \\
& \left.\sum_{\sigma_{2} \in I N C\left(\left.\chi\right|_{V}\right)} \mu_{I N C}\left(\sigma_{2}, 1_{V}\right) \Phi_{\left.\sigma\right|_{V}}\left(a_{l+1}, \cdots, a_{l+s}\right) a_{l+s+1}, \cdots, a_{n}\right) \\
& =\sum_{\substack{\sigma_{1} \leq\left.\pi\right|_{V^{\prime}} \\
\sigma_{1} \in I N C\left(\left.\chi\right|_{V^{\prime}}\right)}} \mu_{I N C}\left(\sigma_{1},\left.\pi\right|_{V^{\prime}}\right) \Phi_{\sigma_{V^{\prime}}}\left(a_{1}, \cdots, a_{l}, \kappa_{\left.\chi\right|_{V}, 1_{V}}\left(a_{l+1}, \cdots, a_{l+s}\right) a_{l+s+1}, \cdots, a_{n}\right) \\
& =\kappa_{\left.\chi\right|_{V^{\prime}},\left.\pi\right|_{V^{\prime}}}\left(a_{1}, \cdots, a_{l}, \kappa_{\left.\chi\right|_{V}, 1_{V}}\left(a_{l+1}, \cdots, a_{l+s}\right) a_{l+s+1}, \cdots, a_{n}\right) .
\end{aligned}
$$

The other part follows from the bi-module property. This finishes the proof.

The preceding theorem shows that $\kappa_{\chi, \pi}\left(a_{1}, \cdots, a_{n}\right)$ is completely determined by cumulant functionals of the form $\kappa_{\chi^{\prime}, 1_{[m]}}$ for $m \in \mathbb{N}$ and $\chi^{\prime}:\{1, \cdots, m\} \rightarrow\{\mathcal{F}, \mathcal{B}\}$.

Definition 4.3. Let $\left\{\left(\mathcal{A}_{i, \mathcal{F}}, \mathcal{A}_{i, \mathcal{B}}\right)\right\}_{i \in \mathcal{I}}$ be a family of pairs of $B$-faces of $\mathcal{A}$ in a $B$-valued probability space $(\mathcal{A}, \mathbb{E})$. We say that the family $\left\{\left(\mathcal{A}_{i, \mathcal{F}}, \mathcal{A}_{i, \mathcal{B}}\right)\right\}_{i \in \mathcal{I}}$ is combinatorially free-Boolean independent with amalgamation over $B$ if

$$
\kappa_{\chi, 1_{n}}\left(a_{1}, \cdots, a_{n}\right)=0
$$

whenever $\omega:\{1, \cdots, n\} \rightarrow \mathcal{I}, \chi:\{1, \cdots, n\} \rightarrow\{\mathcal{F}, \mathcal{B}\}, a_{k} \in \mathcal{A}_{\omega(k), \chi(k)}$ and $\omega$ is not a constant.

Proposition 4.4. Let $\left\{\left(\mathcal{A}_{i, \mathcal{F}}, \mathcal{A}_{i, \mathcal{B}}\right)\right\}_{i \in \mathcal{I}}$ be a family of pairs of $B$-faces in a $B$-valued probability space $(\mathcal{A}, \mathbb{E})$. Then $\kappa_{\chi, 1_{n}}$ has the following additivity property:

$$
\kappa_{\chi, 1_{n}}\left(a_{1,1}+a_{2,1}, \cdots, a_{1, n}+a_{2, n}\right)=\kappa_{\chi, 1_{n}}\left(a_{1,1}, \cdots, a_{1, n}\right)+\kappa_{\chi, 1_{n}}\left(a_{2,1}, \cdots, a_{2, n}\right)
$$

whenever $\omega_{1}, \omega_{2}:[n]=\{1, \cdots, n\} \rightarrow \mathcal{I}, \chi:\{1, \cdots, n\} \rightarrow\{\mathcal{F}, \mathcal{B}\}, a_{1, k} \in \mathcal{A}_{\omega_{1}(k), \chi(k)}, a_{2, k} \in$ $\mathcal{A}_{\omega_{2}(k), \chi(k)}$ and $\omega_{1}([n]) \cap \omega_{2}([n])=\emptyset$.

Proof. By a direct calculation, we have

$$
\kappa_{\chi, 1_{n}}\left(a_{1,1}+a_{2,1}, \cdots, a_{1, n}+a_{2, n}\right)=\sum_{i_{1}, \ldots i_{n} \in\{1,2\}} \kappa_{\chi, 1_{n}}\left(a_{i_{1}, 1}, \cdots, a_{i_{n}, n}\right) .
$$


Since $\left\{\left(\mathcal{A}_{i, \mathcal{F}}, \mathcal{A}_{i, \mathcal{B}}\right)\right\}_{i \in I}$ are combinatorially free-Boolean independent, by Definition 4.3 , we have

$$
\kappa_{\chi, 1_{n}}\left(a_{i_{1}, 1}, \cdots, a_{i_{n}, n}\right)=0
$$

if $i_{j} \neq i_{k}$ for some $j, k \in\{1, \cdots, n\}$. The result follows.

Proposition 4.5. Let $\left\{\left(\mathcal{A}_{i, \mathcal{F}}, \mathcal{A}_{i, \mathcal{B}}\right)\right\}_{i \in \mathcal{I}}$ be a combinatorially free-Boolean independent family of pairs of $B$-faces in a $B$-valued probability space $(\mathcal{A}, \mathbb{E})$. Assume that $\pi=\left\{V_{1}, \cdots, V_{p}\right\} \in$ $\operatorname{INC}(\chi)$. Then

$$
\kappa_{\chi, \pi}\left(a_{1}, \cdots, a_{n}\right)=0
$$

whenever $\omega:\{1, \cdots, n\} \rightarrow \mathcal{I}, \chi:\{1, \cdots, n\} \rightarrow\{\mathcal{F}, \mathcal{B}\}, a_{k} \in \mathcal{A}_{\omega(k), \chi(k)}$ and $\omega$ is not a constant on a block $W$ of $\pi$.

Proof. We prove the statement by induction on the number of blocks of $\pi$.

When $p=1$, then the statement follows from Definition 4.3. Suppose now that $p>1$, let $V=(l+1, l+2, \cdots, l+s)$ be an interval block of $\pi$. By Proposition 4.2, we have

$$
\kappa_{\chi, \pi}\left(a_{1}, \cdots, a_{n}\right)=\kappa_{\left.\chi\right|_{V^{\prime}},\left.\pi\right|_{V^{\prime}}}\left(a_{1}, \cdots, a_{l}, \kappa_{\left.\chi\right|_{V}, 1_{V}}\left(a_{l+1}, \cdots, a_{l+s}\right) a_{l+s+1}, \cdots, a_{n}\right),
$$

where $V^{\prime}=\{1, \cdots, n\} \backslash V$. If $\omega$ is not a constant on $V$, then $\kappa_{\chi, \pi}\left(a_{1}, \cdots, a_{n}\right)=0$. Otherwise, $\left.\omega\right|_{V^{\prime}}$ in not a constant on a block of $\left.\pi\right|_{V^{\prime}}$. The statement follows from an induction argument.

4.2. Free-Boolean independence is equivalent to combinatorially free-Boolean independence. In this subsection, we will prove that free-Boolean independence defined in Definition 2.5 is equivalent to the combinatorially free-Boolean independence given in Definition 4.3 We will show that mixed moments are uniquely determined by lower order mixed moments in the same way for both free-Boolean independence and combinatorially free-Boolean independence.

The proof for following result is essentially the same as the proof of in [11, Proposition 10.6] in free probability context and we thus leave the details to the reader. Applying Theorem 4.2, we have the following result.

Lemma 4.6. Let $\chi:\{1, \cdots, n\} \rightarrow\{\mathcal{F}, \mathcal{B}\}$ and $a_{1}, \cdots, a_{n}$ be noncommutative random variables in a $B$-valued probability space $(\mathcal{A}, \mathbb{E})$. Then

$$
\mathbb{E}\left(a_{1} \cdots a_{n}\right)=\sum_{\pi \in I N C(\chi)} \kappa_{\chi, \pi}\left(a_{1} \cdots a_{n}\right) .
$$

For combinatorially free-Boolean independent random variables, we have the following result.

Lemma 4.7. Let $\left\{\left(\mathcal{A}_{i, \mathcal{F}}, \mathcal{A}_{i, \mathcal{B}}\right)\right\}_{i \in \mathcal{I}}$ be a family of combinatorially free-Boolean independent pairs of $B$-faces in a $B$-valued probability space $(\mathcal{A}, \mathbb{E})$. Assume that $a_{k} \in \mathcal{A}_{\omega(k), \chi(k)}$, where $\omega$ : $\{1, \cdots, n\} \rightarrow \mathcal{I}, \chi:\{1, \cdots, n\} \rightarrow\{\mathcal{F}, \mathcal{B}\}$. Let $\epsilon=\operatorname{ker}(\omega)$. Then,

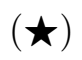

$$
\mathbb{E}\left(a_{1} \cdots a_{n}\right)=\sum_{\sigma \in I N C(\chi)}\left(\sum_{\substack{\pi \in I N C(\chi) \\ \sigma \leq \pi \leq \epsilon}} \mu_{I N C}(\sigma, \pi)\right) \Phi_{\sigma}\left(a_{1} \cdots a_{n}\right) .
$$


Proof. By Lemma 4.6, we have

$$
\mathbb{E}\left(a_{1} \cdots a_{n}\right)=\sum_{\pi \in I N C(\chi)} \kappa_{\chi, \pi}\left(a_{1} \cdots a_{n}\right) .
$$

For each $\pi \in I N C(\chi)$, write its blocks as $\pi=\left\{V_{1}, \cdots, V_{p}\right\}$. Since $\left\{\left(\mathcal{A}_{i, \mathcal{F}}, \mathcal{A}_{i, r}\right)\right\}_{i \in \mathcal{I}}$ are combinatorially free-Boolean independent, by Lemma 4.5, we have

$$
\kappa_{\chi, \pi}\left(a_{1} \cdots a_{n}\right)=0
$$

if $\omega$ is not a constant on some block $V_{s}$ of $\pi$. In other words, $\kappa_{\chi, \pi}\left(a_{1}, \cdots, a_{n}\right) \neq 0$ only if $\omega$ is a constant on $V_{s}$ for all $s$, which implies that $V_{s}$ is contained in a block of $\epsilon$ for all $s$, i.e., $\pi \leq \epsilon$. Therefore, we have

$$
\begin{aligned}
\mathbb{E}\left(a_{1} \cdots a_{n}\right) & =\sum_{\pi \in I N C(\chi), \pi \leq \epsilon} \kappa_{\chi, \pi}\left(a_{1}, \cdots, a_{n}\right) \\
& =\sum_{\pi \in I N C(\chi), \pi \leq \epsilon}\left(\sum_{\substack{\sigma \in I N C(\chi) \\
\sigma \leq \pi}} \mu_{I N C}(\sigma, \pi) \Phi_{\sigma}\left(a_{1}, \cdots, a_{n}\right)\right) \\
& =\sum_{\sigma \in I N C(\chi)}\left(\sum_{\substack{\pi \in I N C(\chi) \\
\sigma \leq \pi \leq \epsilon}} \mu_{I N C}(\sigma, \pi)\right) \Phi_{\sigma}\left(a_{1}, \cdots, a_{n}\right) .
\end{aligned}
$$

This finishes the proof.

We now turn to consider the case that the family $\left\{\left(\mathcal{A}_{i, \mathcal{F}}, \mathcal{A}_{i, \mathcal{B}}\right)\right\}_{i \in \mathcal{I}}$ is free-Boolean independent in $(\mathcal{A}, B, \mathbb{E})$ in the sense of Definition 2.5. In what follows, we assume that $a_{k} \in \mathcal{A}_{\omega(k), \chi(k)}$, where $\omega:\{1, \cdots, n\} \rightarrow \mathcal{I}, \chi:\{1, \cdots, n\} \rightarrow\{\mathcal{F}, \mathcal{B}\}$. Let $\epsilon=\operatorname{ker}(\omega)$, the kernel of $\omega$. Recall that $\chi^{-1}(\mathcal{B}) \cap[2, n-1]=\left\{l_{1}, \cdots, l_{m-1}\right\}$. Let $\chi_{1}\left(\right.$ or $\left.\epsilon_{1}\right)$ be the restriction of $\chi$ (or $\left.\epsilon\right)$ to $\left\{1, \cdots, l_{1}\right\}$ respectively. Let $\chi_{1}^{\prime}$ (or $\epsilon_{1}^{\prime}$ ) be the restriction of $\chi$ (or $\epsilon$ ) to the interval $\left\{l_{1}, \cdots, n\right\}$ respectively. We need to show that the the mixed moments $\mathbb{E}\left(a_{1} \cdots a_{n}\right)$ can be determined in the same way as in Lemma 4.7 .

To this end, it is enough to consider the case that $\mathcal{A}=\mathcal{L}(\mathcal{X}), \mathcal{A}_{i, \mathcal{F}}=\lambda_{i}\left(\mathcal{L}\left(\mathcal{X}_{i}\right)\right)$ and $\mathcal{A}_{i, \mathcal{B}}=$ $P_{i} \lambda_{i}\left(\mathcal{L}\left(\mathcal{X}_{i}\right)\right) P_{i}$, where $\left\{\left(\mathcal{X}_{i}, \dot{\mathcal{X}}_{i}, p_{i}\right)\right\}_{i \in I}$ is a family of vector spaces with specified vectors and $(\mathcal{X}, \dot{\mathcal{X}}, p)$ is the reduced free product of them.

We will prove the mixed moments formula $(\star)$ in Lemma 4.7 by induction on the number of elements of $\chi^{-1}(\mathcal{B}) \cap[2, n-1]$.

Lemma 4.8. If $\chi(n)=\mathcal{B}$, then there exists an operator $T \in \mathcal{A}_{\omega(n), \mathcal{F}}$ such that

$$
\mathbb{E}\left(a_{1} \cdots a_{n}\right)=\mathbb{E}\left(a_{1} \cdots a_{n-1} T\right) .
$$

Proof. If $n \in \chi^{-1}(\mathcal{B})$, then $a_{n} \in \mathcal{A}_{\omega(n), \mathcal{B}}=P_{\omega(n)} \lambda_{\omega(n)}\left(\mathcal{L}\left(\mathcal{X}_{\omega(n)}\right)\right) P_{\omega(n)}$. Assume that $a_{n}=$ $P_{\omega(n)} T P_{\omega(n)}$ for some $T \in \lambda_{\omega(n)}\left(\mathcal{L}\left(\mathcal{X}_{\omega(n)}\right)\right)$. Then

$$
a_{1} \cdots a_{n} 1_{B}=a_{1} \cdots a_{n-1} P_{\omega(n)} T P_{\omega(n)} 1_{B}=a_{1} \cdots T 1_{B}=a_{1} \cdots a_{n-1} T 1_{B}
$$

since $T 1_{B} \in P_{\omega(n)} \mathcal{X}$. Thus, the mixed moments are the same if we replace $a_{n}$ by the element $T \in \lambda_{\omega(n)}\left(\mathcal{L}\left(\mathcal{X}_{\omega(n)}\right)\right)$. 
Lemma 4.9. If $\chi(1)=\mathcal{B}$, then there exists an operator $T \in \mathcal{A}_{\omega(n), \mathcal{F}}$ such that

$$
\mathbb{E}\left(a_{1} \cdots a_{n}\right)=\mathbb{E}\left(T a_{2} \cdots a_{n}\right) .
$$

Proof. If $1 \in \chi^{-1}(\mathcal{B})$, then $a_{1} \in \mathcal{A}_{\omega(1), \mathcal{B}}=P_{\omega(1)} \lambda_{\omega(1)}\left(\mathcal{L}\left(\mathcal{X}_{\omega(1)}\right)\right) P_{\omega(1)}$. Assume that $a_{1}=$ $P_{\omega(1)} T P_{\omega(1)}$ for some $T \in \lambda_{\omega(1)}\left(\mathcal{L}\left(\mathcal{X}_{\omega(1)}\right)\right)$. Recall that $p$ is the projection $p: \mathcal{X} \rightarrow B$. Notice that $p P_{\omega(1)}=p$ and

$$
\begin{aligned}
\mathbb{E}\left(a_{1} \cdots a_{n}\right) & =p P_{\omega(1)} T P_{\omega(1)} a_{2} \cdots a_{n} 1_{B} \\
& =p P_{\omega(1)} T P_{\omega(1)} a_{2} \cdots a_{n} 1_{B} \\
& =p T P_{\omega(1)} a_{2} \cdots a_{n} 1_{B} \\
& =p T a_{2} \cdots a_{n} 1_{B}-p T\left(1_{\mathcal{X}}-P_{\omega(1)}\right) a_{2} \cdots a_{n} 1_{B}
\end{aligned}
$$

where $1_{\mathcal{X}}$ is the identity operator in $\mathcal{L}(\mathcal{X})$. Notice that

$$
\left(1_{\mathcal{X}}-P_{\omega(1)}\right) a_{2} \cdots a_{n} 1_{B} \in \bigoplus_{i \neq \omega(1)} \dot{\mathcal{X}}_{i} \oplus \bigoplus_{n \geq 2}\left(\bigoplus_{i_{1} \neq i_{2} \neq \cdots \neq i_{n}} \dot{\mathcal{X}}_{i_{1}} \otimes_{B} \cdots \otimes_{B} \dot{\mathcal{X}}_{i_{n}}\right)
$$

and

$$
\begin{aligned}
\bigoplus_{i \neq \omega(1)} \dot{\mathcal{X}}_{i} \oplus \bigoplus_{n \geq 2}\left(\bigoplus_{i_{1} \neq i_{2} \neq \cdots \neq i_{n}}^{\bigoplus} \dot{\mathcal{X}}_{i_{1}} \otimes_{B} \cdots \otimes_{B} \dot{\mathcal{X}}_{i_{n}}\right) \\
=V_{\omega(1)}\left(\mathcal{X}_{\omega(1)} \otimes_{B}(\underbrace{}_{\substack{\bigoplus_{i_{1} \neq i_{2} \neq \cdots \neq i_{n}} \\
i_{1} \neq \omega(1), n \geq 1}} \dot{\mathcal{X}}_{i_{1}} \otimes_{B} \cdots \otimes_{B} \dot{\mathcal{X}}_{i_{n}})\right) .
\end{aligned}
$$

Therefore, $\bigoplus_{i \neq \omega(1)} \dot{\mathcal{X}}_{i} \oplus \bigoplus_{n \geq 2}\left(\bigoplus_{i_{1} \neq i_{2} \neq \cdots \neq i_{n}} \dot{\mathcal{X}}_{i_{1}} \otimes_{B} \cdots \otimes_{B} \dot{\mathcal{X}}_{i_{n}}\right)$ is an invariant subspace of $T$ and

$$
\left.p T\left(I_{\mathcal{X}}-P_{\omega(1)}\right) a_{2} \cdots a_{n} 1_{B}=p\left(I_{\mathcal{X}}-P_{\omega(1)}\right) T\left(I_{\mathcal{X}}-P_{\omega(1)}\right) a_{2} \cdots a_{n} 1_{B}\right)=0,
$$

where the last equality follows from the fact that $p\left(I_{\mathcal{X}}-P_{\omega(1)}\right)=0$. We thus proved that the mixed moments $\mathbb{E}\left(a_{1} \cdots a_{n}\right)$ will be the same if we replace $a_{1}$ by the element $T \in \lambda_{\omega(1)}\left(\mathcal{L}\left(\mathcal{X}_{\omega(1)}\right)\right)$.

We start with the following result.

Lemma 4.10. When $\left|\chi^{-1}(\mathcal{B}) \cap[2, n-1]\right|=0$, we have

$$
\mathbb{E}\left(a_{1} \cdots a_{n}\right)=\sum_{\sigma \in I N C(\chi)}\left(\sum_{\substack{\pi \in I N C(\chi) \\ \sigma \leq \pi \leq \epsilon}} \mu_{I N C}(\sigma, \pi)\right) \Phi_{\sigma}\left(a_{1} \cdots a_{n}\right) .
$$

Proof. By Lemma 4.8 and 4.9 , if $a_{1} \in \mathcal{A}_{\omega(1), \mathcal{B}}$ or $a_{n} \in \mathcal{A}_{\omega(n), \mathcal{B}}$, we may replace $a_{1}$ by $T_{1} \in \mathcal{A}_{\omega(1), \mathcal{F}}$ and $a_{n}$ by $T_{2} \in \mathcal{A}_{\omega(n), \mathcal{F}}$, we still have

$$
\Phi\left(a_{1} \cdots a_{n}\right)=\Phi\left(T_{1} a_{2} \cdots a_{n-1} T_{2}\right) .
$$


Hence, when $\left|\chi^{-1}(\mathcal{B}) \cap[2, n-1]\right|=0$, we may assume that $T_{1}, a_{2}, \cdots, a_{n-1}, T_{2}$ are from the left faces of algebras $\mathcal{A}_{\omega(k), \mathcal{F}}$. Notice that the family $\left\{\left(\mathcal{A}_{i, \mathcal{F}}\right)\right\}_{i \in \mathcal{I}}$ is freely independent with amalgamation in $(\mathcal{A}, \mathbb{E})$ (see [11, 16]), we have

$$
\begin{aligned}
\mathbb{E}\left(a_{1} \cdots a_{n}\right) & =\mathbb{E}\left(T_{1} a_{2} \cdots a_{n-1} T_{2}\right) \\
& =\sum_{\pi \in N C(n), \pi \leq \epsilon} \kappa_{\pi}\left(T_{1}, a_{2}, \cdots, a_{n-1}, T_{2}\right) \\
& =\sum_{\sigma \in N C(n)}\left(\sum_{\substack{\pi \in N C(n) \\
\sigma \leq \pi \leq \epsilon}} \mu(\sigma, \pi)\right) \Phi_{\sigma}\left(T_{1}, a_{2}, \cdots, a_{n-1}, T_{2}\right) \\
& =\sum_{\sigma \in I N C(\chi)}\left(\sum_{\substack{\pi \in I N C(\chi) \\
\sigma \leq \pi \leq \epsilon}} \mu_{I N C}(\sigma, \pi)\right) \Phi_{\sigma}\left(T_{1}, a_{2}, \cdots, a_{n-1}, T_{2}\right) \\
& =\sum_{\sigma \in I N C(\chi)}\left(\sum_{\substack{\pi \in I N C(\chi) \\
\sigma \leq \pi \leq \epsilon}} \mu_{I N C}(\sigma, \pi)\right) \Phi_{\sigma}\left(a_{1}, a_{2}, \cdots, a_{n-1}, a_{2}\right),
\end{aligned}
$$

where we used the fact that $\operatorname{INC}(\chi)=N C(n)$ when $\left|\chi^{-1}(\mathcal{B}) \cap[2, n-1]\right|=0$.

Now, we are now ready to prove our main theorem.

Theorem 4.11. Let $\left\{\left(\mathcal{A}_{i, \mathcal{F}}, \mathcal{A}_{i, \mathcal{B}}\right)\right\}_{i \in I}$ be a family of pairs of $B$-faces in a $B$-valued probability space $(\mathcal{A}, \mathbb{E})$. The family $\left\{\left(\mathcal{A}_{i, \mathcal{F}}, \mathcal{A}_{i, \mathcal{B}}\right)\right\}_{i \in \mathcal{I}}$ is free-Boolean independent with amalgamation over $B$ if and only if they are combinatorially free-Boolean independent with amalgamation over $B$.

Proof. It suffices to show that Equation ( $\star$ ) holds by assuming that $\left\{\left(\mathcal{A}_{i, \mathcal{F}}, \mathcal{A}_{i, \mathcal{B}}\right)\right\}_{i \in \mathcal{I}}$ is freeBoolean independent with amalgamation over $B$. When $\left|\chi^{-1}(\mathcal{B}) \cap[2, n-1]\right|=0$, it is Lemma 4.10. Assume now that Equation $(\star)$ in Lemma 4.7 holds whenever $\left|\chi^{-1}(\mathcal{B}) \cap[2, n-1]\right| \leq m-2$. We shall prove it holds when $\left|\chi^{-1}(\mathcal{B}) \cap[2, n-1]\right|=m-1$. Set $\chi^{-1}(\mathcal{B})=\left\{l_{1}<\cdots<l_{m-1}\right\}$ and $l_{0}=1, l_{m}=n$.

Let $A_{1}=\prod_{i=l_{1}}^{n} a_{i}$. Then $A_{1}\left(1_{B}\right) \in B \oplus \dot{\mathcal{X}}_{\omega\left(l_{1}\right)}$. Since the range of $A_{l_{1}}$ is $B \oplus \dot{\mathcal{X}}_{\omega\left(l_{1}\right)}$, we can view $A_{1}: B \oplus \dot{\mathcal{X}}_{\omega\left(l_{1}\right)} \rightarrow B \oplus \dot{\mathcal{X}}_{\omega\left(l_{1}\right)}$ as a linear operator. In this way, $A_{1}$ is considered as an element in $\lambda_{\omega\left(l_{1}\right)}\left(\mathcal{L}\left(\mathcal{X}_{\omega\left(l_{1}\right)}\right)\right)$

Apply the induction for the $l_{1}$-tuple $\left(a_{1}, \cdots, a_{l_{1}-1}, A_{1}\right)$. Recall that $\epsilon_{1}$ is the restriction of $\epsilon$ to the interval $\left[1, l_{1}\right]$, we have

$$
\begin{aligned}
\mathbb{E}\left(a_{1} \cdots a_{n}\right) & =\mathbb{E}\left(a_{1} \cdots a_{l_{1}-1} A_{1}\right) \\
& =\sum_{\substack{\sigma_{1} \in N C\left(\left[l_{1}\right]\right) \\
\sigma_{1} \leq \epsilon_{1}}}\left(\sum_{\substack{\pi_{1} \in N C\left(\left[l_{1}\right]\right) \\
\sigma_{1} \leq \pi_{1} \leq \epsilon_{1}}} \mu\left(\sigma_{1}, \pi_{1}\right)\right) \Phi_{\sigma_{1}}\left(a_{1}, \cdots, a_{l_{1}-1} A_{1}\right) .
\end{aligned}
$$

We now fix $\sigma_{1} \in N C\left(l_{1}\right), \sigma_{1} \leq \epsilon_{1}$. We shall express $\Phi_{\sigma_{1}}\left(a_{1}, \cdots, a_{l_{1}-1} A_{1}\right)$ according to the definition given by (11). We need to know how $\Phi_{\sigma_{1}}$ is decomposed. To this end, suppose that $V$ is 
the block of $\sigma_{1}$ which contains the element $l_{1}$. Denote that $V=\left\{p_{1}, p_{2}, \cdots, p_{k_{1}}\right\}$, where $p_{k_{1}}=l_{1}$. Set $W_{1}=\left[1, p_{1}-1\right], W_{2}=\left[p_{1}+1, p_{2}-1\right], \cdots, W_{k_{1}}=\left[p_{k_{1}-1}+1, p_{k_{1}}-1\right]=\left[p_{k_{1}-1}+1, l_{1}-1\right]$ ( $W_{i}$ will be the empty set if $p_{i-1}+1=p_{i}$ ), as illustrated in the picture below.

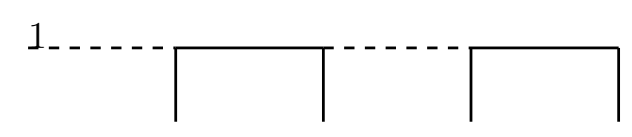

Note that $l_{1} \notin W_{i}$ for all $1 \leq i \leq k_{1}$, we have

$$
\begin{aligned}
& \Phi_{\sigma_{1}}\left(a_{1}, \cdots, a_{l_{1}-1}, A_{1}\right) \\
= & \mathbb{E}\left\{\left(\prod_{k=1}^{k_{1}-1}\left[\left.\Phi_{\left.\sigma_{1}\right|_{W_{k}}}\left(a_{1}, \cdots, a_{l_{1}-1}, A_{1}\right)\right|_{W_{k}}\right] a_{p_{k}}\right)\left[\left.\Phi_{\left.\sigma_{1}\right|_{W_{k_{1}}}}\left(a_{1}, \cdots, a_{l_{1}-1}, A_{1}\right)\right|_{W_{k_{1}}}\right] A_{1}\right\} \\
= & \mathbb{E}\left\{\left(\prod_{k=1}^{k_{1}-1}\left[\left.\Phi_{\left.\sigma_{1}\right|_{W_{k}}}\left(a_{1}, \cdots, a_{l_{1}-1}\right)\right|_{W_{k}}\right] a_{p_{k}}\right)\left[\left.\Phi_{\left.\sigma_{1}\right|_{W_{k_{1}}}}\left(a_{1}, \cdots, a_{l_{1}-1}\right)\right|_{W_{k_{1}}}\right] A_{1}\right\} \\
= & \mathbb{E}\left\{\left(\prod_{k=1}^{k_{1}-1}\left[\left.\Phi_{\left.\sigma_{1}\right|_{W_{k}}}\left(a_{1}, \cdots, a_{l_{1}-1}\right)\right|_{W_{k}}\right] a_{p_{k}}\right)\left[\left.\Phi_{\left.\sigma_{1}\right|_{W_{k_{1}}}}\left(a_{1}, \cdots, a_{l_{1}-1}\right)\right|_{W_{k_{1}}}\right] a_{l_{1}} \cdots a_{m}\right\} .
\end{aligned}
$$

Denote by $A_{2}=\left(\prod_{k=1}^{k_{1}-1}\left[\left.\Phi_{\left.\sigma_{1}\right|_{W_{k}}}\left(a_{1}, \cdots, a_{l_{1}-1}\right)\right|_{W_{k}}\right] a_{p_{k}}\right)\left[\left.\Phi_{\left.\sigma_{1}\right|_{W_{k_{1}}}}\left(a_{1}, \cdots, a_{l_{1}-1}\right)\right|_{W_{k_{1}}}\right] a_{l_{1}}$. Notice that $\left|\chi^{-1}(\mathcal{B}) \cap\left[l_{1}+1, n-1\right]\right|=m-2$. We now apply the induction formula for the tuple $\left(A_{2}, a_{l_{1}+1}, \cdots, a_{n}\right)$, recall that $\chi^{\prime}$ is the restriction of $\chi$ to the interval $\left[l_{1}, n\right]$ and $\epsilon^{\prime}$ is the restriction of $\epsilon$ to the interval $\left[l_{1}, n\right]$, we deduce that

$$
\begin{aligned}
& \Phi_{\sigma_{1}}\left(a_{1}, \cdots, a_{l_{1}-1}, A_{1}\right)=\mathbb{E}\left(A_{2} a_{l_{1}+1} a_{l_{1}+2} \cdots a_{n}\right) \\
= & \sum_{\sigma^{\prime} \in I N C\left(\chi^{\prime}\right)}\left(\sum_{\substack{\pi^{\prime} \in I N C\left(\chi^{\prime}\right) \\
\sigma^{\prime} \leq \pi^{\prime} \leq \epsilon^{\prime}}} \mu_{I N C}\left(\sigma^{\prime}, \pi^{\prime}\right)\right) \Phi_{\sigma^{\prime}}\left(A_{2}, a_{l_{1}+1}, a_{l_{1}+2}, \cdots, a_{n}\right) .
\end{aligned}
$$

We now fix $\sigma^{\prime} \in I N C\left(\chi^{\prime}\right), \sigma^{\prime} \leq \epsilon^{\prime}$. We need to express $\Phi_{\sigma^{\prime}}\left(A_{2}, a_{l_{1}+1}, a_{l_{1}+2}, \cdots, a_{n}\right)$ according to the definition given in (11). To this end, suppose that $V^{\prime}$ is the block of $\sigma^{\prime}$ which contains the element $l_{1}$. Suppose that $V^{\prime}=\left\{q_{1}, q_{2}, \cdots, q_{k_{2}}\right\}$, where $q_{1}=l_{1}$. Let $W_{1}^{\prime}=\left[l_{1}, q_{2}-1\right]$, $W_{2}^{\prime}=\left[q_{2}+1, q_{3}-1\right], \cdots, W_{k_{2}}^{\prime}=\left[q_{k_{2}}+1, n\right]\left(W_{j}^{\prime}=\emptyset\right.$ if $\left.q_{j}+1=q_{j+1}\right)$, as shown in the picture below

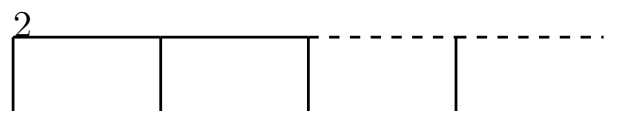

Notice that $l_{1} \notin W_{j}^{\prime}$ for all $1 \leq j \leq q_{k_{2}}$, we apply the induction assumption to the tuple $\left(A_{2}, a_{l_{1}+1}, a_{l_{1}+2}, \cdots, a_{n}\right)$ to obtain the following: 


$$
\begin{aligned}
& \Phi_{\sigma^{\prime}}\left(A_{2}, a_{l_{1}+1}, a_{l_{1}+2}, \cdots, a_{n}\right) \\
= & \mathbb{E}\left\{A_{2}\left[\left.\Phi_{\left.\sigma^{\prime}\right|_{W_{1}^{\prime}}}\left(A_{2}, a_{l_{1}+1}, \cdots, a_{n}\right)\right|_{W_{1}^{\prime}}\right]\left(\prod_{k=2}^{k_{2}} a_{q_{k}}\left[\left.\Phi_{\sigma^{\prime} \mid W_{k}^{\prime}}\left(A_{2}, a_{l_{1}+1}, \cdots, a_{n}\right)\right|_{W_{k}^{\prime}}\right]\right)\right\} \\
= & \mathbb{E}\left\{A_{2}\left[\left.\Phi_{\left.\sigma^{\prime}\right|_{W_{1}^{\prime}}}\left(a_{l_{1}+1}, \cdots, a_{n}\right)\right|_{W_{1}^{\prime}}\right]\left(\prod_{k=2}^{k_{2}} a_{q_{k}}\left[\left.\Phi_{\sigma^{\prime} \mid W_{k}^{\prime}}\left(a_{l_{1}+1}, \cdots, a_{n}\right)\right|_{W_{k}^{\prime}}\right]\right)\right\} \\
= & \mathbb{E}\left\{\left(\prod_{k=1}^{k_{1}-1}\left[\left.\Phi_{\left.\sigma_{1}\right|_{W_{k}}}\left(a_{1}, \cdots, a_{l_{1}-1}\right)\right|_{W_{k}}\right] a_{p_{k}}\right)\left[\left.\Phi_{\left.\sigma_{1}\right|_{W_{k_{1}}}}\left(a_{1}, \cdots, a_{l_{1}-1}\right)\right|_{W_{k_{1}}}\right]\right. \\
& \left.a_{l_{1}}\left[\left.\Phi_{\left.\sigma^{\prime}\right|_{W_{1}^{\prime}}}\left(a_{l_{1}+1}, \cdots, a_{n}\right)\right|_{W_{1}^{\prime}}\right]\left(\prod_{k=2}^{k_{2}} a_{q_{k}}\left[\left.\Phi_{\sigma^{\prime} \mid W_{k}^{\prime}}\left(a_{l_{1}+1}, \cdots, a_{n}\right)\right|_{W_{k}^{\prime}}\right]\right)\right\}
\end{aligned}
$$

Recall that $\alpha_{1}^{\prime}(\pi):=\left(\alpha_{1}(\pi), \alpha^{\prime}(\pi)\right)$ defined in Proposition 3.4, Let $\sigma=\alpha_{1}^{\prime-1}\left(\sigma_{1}, \sigma^{\prime}\right)$. We draw the picture below to show the block $V \in \sigma_{1}$ and the block $V^{\prime} \in \sigma_{2}$ which contain $l_{1}$.

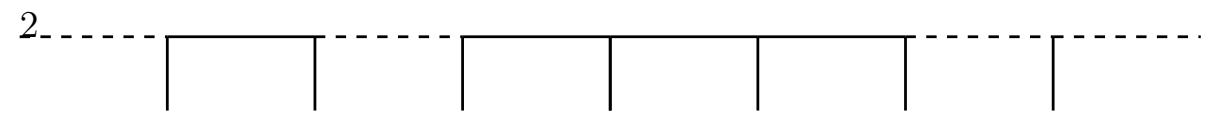

Then,

$$
\begin{aligned}
& \Phi_{\sigma^{\prime}}\left(A_{2}, a_{l_{1}+1}, a_{l_{1}+2}, \cdots, a_{n}\right) \\
= & \mathbb{E}\left\{\left.\left(\prod_{k=1}^{k_{1}-1}\left[\left.\Phi_{\left.\sigma_{1}\right|_{W_{k}}}\left(a_{1}, \cdots, a_{l_{1}-1}\right)\right|_{W_{k}}\right] a_{p_{k}}\right) \Phi_{\left.\sigma_{1}\right|_{W_{k}}}\left(a_{1}, \cdots, a_{l_{1}-1}\right)\right|_{W_{k}}\right. \\
& \left.a_{l_{1}}\left[\Phi_{\left.\sigma^{\prime}\right|_{W_{1}^{\prime}}}\left(a_{l_{1}+1}, \cdots,\left.a_{n}\right|_{W_{1}^{\prime}}\right)\right]\left(\prod_{k=2}^{k_{2}} a_{q_{k}} \Phi_{\left.\sigma^{\prime}\right|_{W_{k}^{\prime}}}\left(a_{l_{1}+1}, \cdots,\left.a_{n}\right|_{W_{k}^{\prime}}\right)\right)\right\} \\
= & \Phi_{\sigma}\left(a_{1}, \cdots, a_{n}\right) .
\end{aligned}
$$

Putting (2), (3), (4), (5) together, we have

$$
\begin{aligned}
& \mathbb{E}\left(a_{1} \cdots a_{n}\right) \\
& =\sum_{\substack{\sigma_{1} \in N C\left(\left[l_{1}\right]\right) \\
\sigma_{1} \leq \epsilon_{1}}}\left(\sum_{\substack{\pi_{1} \in N C\left(\left[l_{1}\right]\right) \\
\sigma_{1} \leq \pi_{1} \leq \epsilon_{1}}} \mu\left(\sigma_{1}, \pi_{1}\right)\right) \Phi_{\sigma_{1}}\left(a_{1}, \cdots, a_{l_{1}-1} A_{1}\right) . \\
& \left.=\sum_{\substack{\sigma_{1} \in N C\left(\left[l_{1}\right]\right) \\
\text { sigma } \leq \epsilon_{1}}}\left(\sum_{\substack{\pi_{1} \in N C\left(\left[l_{1}\right]\right) \\
\sigma_{1} \leq \pi_{1} \leq \epsilon_{1}}} \mu\left(\sigma_{1}, \pi_{1}\right)\right) \sum_{\substack{\sigma^{\prime} \in I N C\left(\chi^{\prime}\right) \\
\sigma_{1} \leq \pi_{1} \leq \epsilon_{1} \\
\pi^{\prime} \in I N C\left(\chi^{\prime}\right) \\
\sigma^{\prime} \leq \pi^{\prime} \leq \epsilon^{\prime}}} \mu_{I N C}\left(\sigma^{\prime}, \pi^{\prime}\right)\right) \Phi_{\sigma^{\prime}}\left(A_{2}, a_{l_{1}+1}, a_{l_{1}+2}, \cdots, a_{n}\right)
\end{aligned}
$$

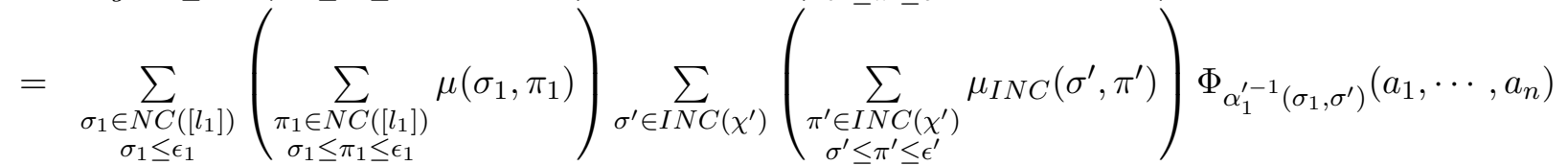

$$
\begin{aligned}
& =\sum_{\sigma \in I N C(\chi)}\left(\sum_{\substack{\pi \in I N C(\chi) \\
\sigma \leq \pi \leq \epsilon}} \mu_{I N C}(\sigma, \pi)\right) \Phi_{\sigma}\left(a_{1}, \cdots, a_{n}\right),
\end{aligned}
$$


where we used Corollary 3.8 in the last identity and thus we obtained our desired equation.

\section{LIMIT THEOREMS}

Let $A=\left(\left(a_{i}\right)_{i \in \mathcal{I}},\left(a_{j}\right)_{j \in \mathcal{J}}\right)$ be a two faced family of noncommutative random variables in a $B$-valued probability space $(\mathcal{A}, \mathbb{E})$. Let $\omega:\{1, \cdots, n\} \rightarrow \mathcal{I} \sqcup \mathcal{J}$ and denote by $\chi_{\omega}:\{1, \cdots, n\} \rightarrow$ $\{\mathcal{F}, \mathcal{B}\}$ the map such that $\chi_{\omega}(k)=\mathcal{B}$ if and only if $\omega(k) \in \mathcal{J}$.

Definition 5.1. A two-faced family $A=\left(\left(a_{i}\right)_{i \in \mathcal{I}},\left(a_{j}\right)_{j \in \mathcal{J}}\right)$ in a $B$-valuede probability space $(\mathcal{A}, \mathbb{E})$ is said to have a centered free-Boolean limit if, for all $n \neq 2$,

$$
\kappa_{\chi \omega, 1_{n}}\left(a_{\omega(1)} b_{1}, \cdots, a_{\omega(n-1)} b_{n-1}, a_{\omega(n)}\right)=0,
$$

for all $\omega:\{1, \cdots, n\} \rightarrow \mathcal{I} \bigsqcup \mathcal{J}$ and $b_{1}, \cdots, b_{n-1} \in B$.

The distribution defined by the the two faced family $A$ is called an operator-valued free-Boolean Gaussian distribution with covariance $C=\left(c_{i, j}\right)_{i, j \in \mathcal{I} \sqcup \mathcal{J}}$, where $C$ is defined by $c_{\omega(1), \omega(2)}(b):=$ $\kappa_{\chi_{\omega}, 1_{2}}\left(a_{\omega(1)}, b a_{\omega(2)}\right)$ for all $\omega:\{1,2\} \rightarrow \mathcal{I} \sqcup \mathcal{J}$ and $b \in B$.

Proposition 5.2. Let $A=\left(\left(a_{i}\right)_{i \in \mathcal{I}},\left(a_{j}\right)_{j \in \mathcal{J}}\right)$ be a two faced family of noncommutative random varialbes in a $B$-valued probability space $(\mathcal{A}, \mathbb{E})$. Let $\omega:\{1,2\} \rightarrow \mathcal{I} \bigsqcup \mathcal{J}$. Then,

$$
\kappa_{\chi_{\omega, 1_{2}}}\left(a_{\omega(1)}, a_{\omega(2)}\right)=\mathbb{E}\left(a_{\omega(1)} a_{\omega(2)}\right)-\mathbb{E}\left(a_{\omega(1)}\right) \mathbb{E}\left(a_{\omega(2)}\right) .
$$

Theorem 5.3. Let $\left\{A_{m}=\left(\left(a_{m, i}\right)_{i \in \mathcal{I}},\left(a_{m, j}\right)_{j \in \mathcal{J}}\right)\right\}_{m=1}^{\infty}$ be a free-Boolean sequence of families in a $B$-valued probability space $(\mathcal{A}, \mathbb{E})$ where $B$ is a Banach space, such that

(1) $\mathbb{E}\left(a_{m, k}\right)=0$ for all $m \in \mathbb{N}$ and $k \in \mathcal{I} \sqcup \mathcal{J}$.

(2) $\sup _{m \in \mathbb{N}}\left\|\mathbb{E}\left(a_{m, \omega(1)} b_{1} \cdots a_{m, \omega(n-1)} b_{n-1} a_{m, \omega(n)}\right)\right\| \leq D_{\omega}<\infty$ for all $n \in \mathbb{N}, \omega:\{1, \cdots, n\} \rightarrow$ $\mathcal{I} \bigsqcup \mathcal{J}$, and $b_{1}, \cdots, b_{n-1} \in B$.

(3) $\lim _{N \rightarrow \infty} N^{-1} \sum_{1 \leq m \leq N} \mathbb{E}\left(a_{m, \omega(1)} b a_{m, \omega(2)}\right)=c_{\omega(1), \omega(2)}(b) \in B$, for all $\omega:\{1,2\} \rightarrow \mathcal{I} \sqcup \mathcal{J}$ and $b \in B$.

Let $S_{N, k}=N^{-1 / 2} \sum_{1 \leq m \leq N} a_{m, k}$ for $k \in \mathcal{I} \sqcup \mathcal{J}$ and $S_{N}=\left(\left(S_{N, i}\right)_{i \in \mathcal{I}},\left(S_{N, j}\right)_{j \in \mathcal{J}}\right)$. Denote by $\gamma_{C}$

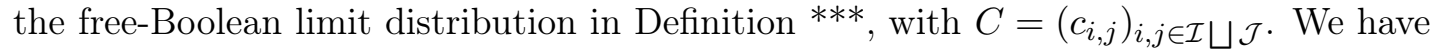

$$
\lim _{N \rightarrow \infty} \mu_{S_{N}}(P)=\gamma_{C}(P)
$$

for all $P \in \mathbb{C}\left\langle a_{k} \mid k \in \mathcal{I} \bigsqcup \mathcal{J}\right\rangle$.

Proof. Since the joint distributions are determined by free-Boolean cumulants uniquely, it is enough to show that

$$
\lim _{N \rightarrow \infty} \kappa_{\chi_{\omega, 1_{n}}}\left(S_{N, \omega(1)} b_{1}, \cdots, S_{N, \omega(n-1)} b_{n-1}, S_{N, \omega(n)}\right)=\kappa_{\chi_{\omega, 1}}\left(S_{\omega(1)} b_{1}, \cdots, S_{\omega(n-1)} b_{n-1}, S_{\omega(n)}\right),
$$

where the two faced family $S=\left(\left(S_{i}\right)_{i \in \mathcal{I}},\left(S_{j}\right)_{j \in \mathcal{J}}\right)$ has a centered $B$-valued free-Boolean Gaussian dsitribution with covariance matrix $C$, for all $n \in \mathbb{N}, \omega:\{1, \cdots, n\} \rightarrow \mathcal{I} \bigsqcup \mathcal{J}$, and $b_{1}, \cdots, b_{n-1} \in$ $B$. 
By the additivity property of free-Boolean cumulants, we have

$$
\begin{aligned}
& \kappa_{\chi \omega, 1_{n}}\left(S_{N, \omega(1)} b_{1}, \cdots, S_{N, \omega(n-1)} b_{n-1}, S_{N, \omega(n)}\right) \\
= & \frac{1}{N^{n / 2}} \sum_{1 \leq m \leq N} \kappa_{\chi_{\omega, 1_{n}}}\left(a_{m, \omega(1)} b_{1}, \cdots, a_{m, \omega(n-1)} b_{n-1}, a_{m, \omega(n)}\right) .
\end{aligned}
$$

Since the free-Boolean cumulant are universal polynomial of mixed moments, we deduce from assumption (2) that

$$
\sup _{m \in \mathbb{N}}\left\|\kappa_{\chi_{\omega, 1_{n}}}\left(a_{m, \omega(1)} b_{1}, \cdots, a_{m, \omega(n-1)} b_{n-1}, a_{m, \omega(n)}\right)\right\|<\infty
$$

and hence

$$
\lim _{N \rightarrow \infty} \kappa_{\chi_{\omega, 1_{n}}}\left(S_{N, \omega(1)} b_{1}, \cdots, S_{N, \omega(n-1)} b_{n-1}, S_{N, \omega(n)}\right)=0
$$

for $n \geq 3$.

As $\kappa_{\chi_{\omega, 1_{1}}}\left(a_{m, \omega(1)}\right)=\mathbb{E}\left(a_{m, \omega(1)}\right)=0$ for all $m \in \mathbb{N}$ and $\omega:\{1\} \rightarrow \mathcal{I} \bigsqcup \mathcal{J}$, we have $\kappa_{\chi_{\omega, 1_{1}}}\left(S_{N, \omega(1)}\right)=$ 0 for all $N \in \mathbb{N}$ and $\omega:\{1\} \rightarrow \mathcal{I} \sqcup \mathcal{J}$. Finally, by assumption (3), and Proposition 5.2, we have

$$
\begin{aligned}
& \kappa_{\chi_{\omega}, 1_{2}}\left(S_{N, \omega(1)} b, S_{N, \omega(2)}\right) \\
= & \frac{1}{N} \sum_{1 \leq m \leq N} \kappa_{\chi_{\omega, 1_{2}}}\left(a_{n, \omega(1)} b, a_{n, \omega(2)}\right) \\
= & \frac{1}{N} \sum_{1 \leq m \leq N} \mathbb{E}\left(a_{n, \omega(1)} b a_{n, \omega(2)}\right) \rightarrow C_{\omega(1), \omega(2)}(b),
\end{aligned}
$$

as $N \rightarrow \infty$, for all $\omega:\{1,2\} \rightarrow \mathcal{I} \sqcup \mathcal{J}$ and $b \in B$. This finishes the proof.

\section{Moment-COnditions for Free-Boolean indePendence}

Let $\left\{\left(\mathcal{X}_{i}, \dot{\mathcal{X}}_{i}, p_{i}\right)\right\}_{i \in \mathcal{I}}$ be $B$-B-bimodules with specified projectin, and $\{\mathcal{X}, \mathcal{X}, p\}$ be the reduced free product with amalgamation over $B$. For each $i \in \mathcal{I}$, denote $\mathcal{A}_{i, \mathcal{F}}=\lambda_{i}\left(\mathcal{L}\left(\mathcal{X}_{i}\right)\right)$ and $\mathcal{A}_{i, \mathcal{B}}=$ $\beta_{i}\left(\mathcal{L}\left(\mathcal{X}_{i}\right)\right)=P_{i} \lambda_{i}\left(\mathcal{L}\left(X_{i}\right)\right) P_{i}$, where $P_{i}$ is the projection onto the subspace $B \oplus \stackrel{\circ}{\mathcal{X}}_{i}$. We also denote by $\mathcal{A}_{i}$ the algebra generated by $\mathcal{A}_{i, \mathcal{F}} \cup \mathcal{A}_{i, \mathcal{B}}$.

Given a family $\left\{C_{i}, D_{i}\right\}_{i \in \mathcal{I}}$ of free-Boolean pair of $B$-faces in a $B$-valued probability space $(\mathcal{A}, \mathbb{E})$, to study the mixed moments of the family, one can identify $C_{i}$ with $\lambda_{i}\left(\gamma_{i}\left(C_{i}\right)\right)$ and identify $D_{i}$ with $\beta_{i}\left(\delta_{i}\left(D_{i}\right)\right)$ following Definition 2.5. In this way, we regard $C_{i}$ as a subalgebra of $\mathcal{A}_{i, \mathcal{F}}$ and $D_{i}$ as a subalgebra of $\mathcal{A}_{i, \mathcal{B}}$ throughout this section.

Definition 6.1. Given a set $S_{i} \subset \mathcal{A}_{i, \mathcal{F}} \cup \mathcal{A}_{i, \mathcal{B}}$ and $a_{1}, \cdots, a_{m} \in S_{i}$ their product $A=a_{1} \cdots a_{m}$ is called a simple product of elements from $S_{i}$. It is called a Boolean product of elements from $S_{i}$ if $a_{k} \in S_{i} \cap \mathcal{A}_{i, \mathcal{B}}$ for some $1 \leq k \leq m$.

If $A=a_{1} \cdots a_{m}$ is a simple product, but not a Boolean product, then each $a_{i} \in S_{i} \cap \mathcal{A}_{i, \mathcal{F}}$. A Boolean product of elements from $\mathcal{A}_{i, \mathcal{F}} \cup \mathcal{A}_{i, \mathcal{B}}$ has a very simple form.

Proposition 6.2. Let $A \in \mathcal{A}_{i}(i \in \mathcal{I})$ be a Boolean product of elements from $\mathcal{A}_{i, \mathcal{F}} \cup \mathcal{A}_{i, \mathcal{B}}$. Then $A \in \mathcal{A}_{i, \mathcal{B}}$, in particular, $A(\mathcal{X}) \subset \mathcal{X}_{i}$. 
Proof. Write $A=a_{1} \cdots a_{m}$ where $a_{k} \in \mathcal{A}_{i, \mathcal{F}} \cup \mathcal{A}_{i, \mathcal{B}}(1 \leq k \leq m)$. For an element $a_{k} \in \mathcal{A}_{i, \mathcal{B}}$, it can be written as $a_{k}=P_{i} a_{k}^{\prime} P_{i}$, where $a_{k}^{\prime} \in \mathcal{A}_{k, \mathcal{F}}$ by definition. Hence $A=P_{i} b_{1} \cdots b_{m} P_{i}$, where

$$
b_{k}=\left\{\begin{array}{lll}
a_{k}^{\prime}, & \text { if } & a_{k} \in \mathcal{A}_{i, \mathcal{B}} \\
a_{k}, & \text { if } & a_{k} \in \mathcal{A}_{i, \mathcal{F}}
\end{array}\right.
$$

by Proposition 2.2. The assertion follows.

To state our result in general, from now on, we will let $S_{i} \subset \mathcal{A}_{i, \mathcal{F}} \cup \mathcal{A}_{i, \mathcal{B}}$ for each $i \in \mathcal{I}$. Typically, $S_{i}=C_{i} \cup D_{i}$ or $S_{i}=\mathcal{A}_{i, \mathcal{F}} \cup \mathcal{A}_{i, \mathcal{B}}$.

Lemma 6.3. Let $A_{1} \in \mathcal{A}_{i}$ and $A_{2} \in \mathcal{A}_{j}$ be two simple product of elements from $S_{i}$ and $S_{j}$ respectively and $i \neq j$. If $A_{1}$ is a Boolean product, then

$$
A_{1} A_{2} 1_{B}=A_{1} \mathbb{E}_{\mathcal{L}(\mathcal{X})}\left(A_{2}\right) .
$$

Proof. Write $A_{2}\left(1_{B}\right)=\mathbb{E}_{\mathcal{L}(\mathcal{X})}\left(A_{2}\right) \oplus \stackrel{\circ}{A}_{2}$, where $\AA_{2} \in \stackrel{\circ}{\mathcal{X}}_{j}$. As $A_{1}$ is a Boolean product, at least one of the factor is from $\mathcal{A}_{i, \mathcal{B}}$, we thus can write $A_{1}=a_{1} b a_{2}$, where $a_{2}$ is a simple product of elements from $\mathcal{A}_{i, \mathcal{F}}$ and $b \in \mathcal{A}_{i, \mathcal{B}}$. We can express $a_{2}=\lambda_{i}(T)$, where $T \in \mathcal{L}_{B}\left(\mathcal{X}_{i}\right)$.

Observe that

$$
\begin{aligned}
\lambda_{i}(T)\left(\AA_{2}\right) & =V_{i}\left(T \otimes I_{\mathcal{X}(i)}\right) V_{i}^{-1} \AA_{2} \\
& =V_{i}\left(T \otimes I_{\mathcal{X}(i)}\right)\left(1_{B} \otimes \AA_{2}\right) \\
& =V_{i}\left[\mathbb{E}_{\mathcal{L}\left(X_{i}\right)}(T) \otimes \AA_{2}+\left(T-\mathbb{E}_{\mathcal{L}\left(\mathcal{X}_{i}\right)}(T)\right) \otimes \AA_{2}\right] .
\end{aligned}
$$

Hence, $\lambda_{i}(T) \AA_{2} \in \dot{\mathcal{X}}_{j} \oplus\left(\dot{\mathcal{X}}_{i} \otimes \dot{\mathcal{X}}_{j}\right)$. As $b=P_{i} b P_{i}$, where $P_{i}$ is the projection onto $B \oplus \dot{\mathcal{X}}_{i}$, we deduce that $b \AA_{2}=0$. We then have

$$
\begin{aligned}
A_{1} A_{2} 1_{B} & =\left(a_{1} b a_{2}\right)\left(\mathbb{E}_{\mathcal{L}(\mathcal{X})}\left(A_{2}\right)+\AA_{2}\right) \\
& =\left(a_{1} b a_{2}\right)\left(\mathbb{E}_{\mathcal{L}(\mathcal{X})}\left(A_{2}\right)\right) \\
& =A_{1}\left(\mathbb{E}_{\mathcal{L}(\mathcal{X})}\left(A_{2}\right)\right) .
\end{aligned}
$$

This finishes the proof.

An application of the preceeding lemman and the bimodule property of expection $\mathbb{E}_{\mathcal{L}(\mathcal{X})}$ implies the following result.

Corollary 6.4. For $1 \leq i \leq m$, let $A_{i} \in \mathcal{A}_{k(i)}$ be Boolean product of elements from $\mathcal{A}_{k(i), \mathcal{F}} \cup$ $\mathcal{A}_{k(i), \mathcal{B}}$ and $k(1) \neq k(2) \neq \cdots \neq k(m)$. Then, we have

$$
\mathbb{E}_{\mathcal{L}(\mathcal{X})}\left(A_{1} \cdots A_{m}\right)=\mathbb{E}_{\mathcal{L}(\mathcal{X})}\left(A_{1}\right) \cdots \mathbb{E}_{\mathcal{L}(\mathcal{X})}\left(A_{m}\right) .
$$

Lemma 6.5. Let $B \in \mathcal{A}_{i}$ be a Boolean product of elements from $S_{i} \subset \mathcal{A}_{i, \mathcal{F}} \cup \mathcal{A}_{i, \mathcal{B}}$, then $B(\mathcal{X}) \subset$ $\mathcal{X}_{i}$

Proof. Observe that $B$ can be written as $B=a_{1} b a_{2}$, where $b \in \mathcal{A}_{i, \mathcal{B}}$ and $a_{1}, a_{2}$ are simple products of elements from $\mathcal{A}_{i, \mathcal{F}} \cup \mathcal{A}_{i, \mathcal{B}}$. Since $b=P_{i} b P_{i}$, where $P_{i}$ is the projection onto $\mathcal{X}_{i}=B \oplus \dot{\mathcal{X}}_{i}$, we deduce that $b a_{2}(\mathcal{X}) \subset \dot{\mathcal{X}}_{i}$. Hence the assertion follows. 
Lemma 6.6. Let $A_{i} \in \mathcal{A}_{k(i)}(1 \leq i \leq m)$ be simple products of elements from $S_{i} \subset \mathcal{A}_{k(i), \mathcal{F}} \cup$ $\mathcal{A}_{k(i), \mathcal{B}}$. If the following conditions hold:

(1) There exist $1<l_{1}<l_{2}<m$ such that $A_{l_{1}}, A_{l_{1}+1}, \cdots, A_{l_{2}}$ are not Boolean products.

(2) $A_{l_{1}-1}$ and $A_{l_{2}+1}$ are Boolean products.

(3) $k(1) \neq k(2) \neq \cdots \neq k(m)$.

(4) $\mathbb{E}_{\mathcal{L}(\mathcal{X})}\left(A_{l_{1}}\right)=\cdots=\mathbb{E}_{\mathcal{L}(\mathcal{X})}\left(A_{l_{2}}\right)=0$.

Then, the product of operators $A_{1} \cdots A_{m}=0$.

Proof. Since $A_{l_{2}+1}$ is a Boolean product, it follows from Lemma 6.5 that

$$
A_{l_{2}+1} \cdots A_{m}(\mathcal{X}) \subset B \oplus{\stackrel{\circ}{\mathcal{X}_{\left(l_{2}+1\right)}}}
$$

As $\mathbb{E}_{\mathcal{L}(X)}\left(A_{l_{1}}\right)=\cdots=\mathbb{E}_{\mathcal{L}(X)}\left(A_{l_{2}}\right)=0$ and each $A_{j}\left(l_{1} \leq j \leq l_{2}\right)$ is a simple product of elements from $\mathcal{A}_{k(j), \mathcal{F}}$, in this case, we then have

$$
\begin{aligned}
A_{l_{2}}\left(A_{l_{2}+1} \cdots A_{m}\right)(\mathcal{X}) & \subset \dot{\mathcal{X}}_{k\left(l_{2}\right)} \otimes\left(B \oplus \stackrel{\circ}{\mathcal{X}}_{k\left(l_{2}+1\right)}\right) \\
& \cong \dot{\mathcal{X}}_{k\left(l_{2}\right)} \oplus\left(\stackrel{\circ}{\mathcal{X}}_{k\left(l_{2}\right)} \otimes \dot{\circ}_{k\left(l_{2}+1\right)}\right) .
\end{aligned}
$$

By induction, we then have

$$
\begin{aligned}
& \left(A_{l_{1}} \cdots A_{l_{2}}\right)\left(A_{l_{2}+1} \cdots A_{m}\right)(\mathcal{X}) \\
& \quad \subset\left(\dot{\mathcal{X}}_{k\left(l_{1}\right)} \otimes \cdots \otimes \dot{\mathcal{X}}_{k\left(l_{2}\right)}\right) \oplus\left(\dot{\mathcal{X}}_{k\left(l_{1}\right)} \otimes \cdots \otimes \dot{\mathcal{X}}_{k\left(l_{2}\right)} \otimes \dot{\mathcal{X}}_{k\left(l_{2}+1\right)}\right) .
\end{aligned}
$$

The operator $A_{l_{1}-1}$ is a Boolean product. We may write $A_{l_{1}-1}=a_{1} b a_{2}$ where $a_{2}$ is a simple product of elements from $\mathcal{A}_{k\left(l_{1}-1\right), \mathcal{F}}$ and $b \in \mathcal{A}_{k\left(l_{1}-1\right), \mathcal{B}}$. If follows that

$$
\begin{aligned}
a_{2}\left(A_{l_{1}} \cdots A_{m}\right)(\mathcal{X}) & \subset \mathcal{X}_{k\left(l_{1}-1\right)} \otimes\left[\left(\dot{\mathcal{X}}_{k\left(l_{1}\right)} \otimes \cdots \otimes \dot{\mathcal{X}}_{k\left(l_{2}\right)}\right) \oplus\left(\dot{\mathcal{X}}_{k\left(l_{1}\right)} \otimes \cdots \otimes \dot{\mathcal{X}}_{k\left(l_{2}\right)} \otimes \dot{\mathcal{X}}_{k\left(l_{2}+1\right)}\right)\right] \\
& \cong\left(\dot{\mathcal{X}}_{k\left(l_{1}\right)} \otimes \cdots \otimes \dot{\mathcal{X}}_{k\left(l_{2}\right)}\right) \oplus\left(\dot{\mathcal{X}}_{k\left(l_{1}\right)} \otimes \cdots \otimes \dot{\mathcal{X}}_{k\left(l_{2}\right)} \otimes \dot{\mathcal{X}}_{k\left(l_{2}+1\right)}\right) \\
& \oplus\left(\dot{\circ}_{k\left(l_{1}-1\right)} \otimes \dot{\mathcal{X}}_{k\left(l_{1}\right)} \otimes \cdots \otimes \dot{\mathcal{X}}_{k\left(l_{2}\right)}\right) \oplus\left(\dot{\mathcal{X}}_{k\left(l_{1}-1\right)} \otimes \dot{\circ}_{k\left(l_{1}\right)} \otimes \cdots \otimes \dot{\mathcal{X}}_{k\left(l_{2}\right)} \otimes \dot{\mathcal{X}}_{k\left(l_{2}+1\right)}\right) .
\end{aligned}
$$

As $b=P_{k\left(l_{1}-1\right)} b P_{k\left(l_{1}-1\right)}$, where $P_{k\left(l_{1}-1\right)}$ is the projection onto $B \oplus \dot{\mathcal{X}}_{k\left(l_{1}-1\right)}$. We then have

$$
b a_{2}\left(A_{l_{1}} \cdots A_{m}\right)(x)=0 \quad \text { for all } x \in \mathcal{X} .
$$

which implies that $A_{1} \cdots A_{m}=0$. This finishes the proof.

Remark 6.7. When $S_{i}=\mathcal{A}_{i, \mathcal{F}} \cup \mathcal{A}_{i, \mathcal{B}}$, note that $\mathcal{A}_{i, \mathcal{B}}=P_{i} \mathcal{A}_{i, \mathcal{F}} P_{i}$ and Proposition 2.2, the Boolean product $A_{l_{1}-1}$ can be written as $A_{l_{1}-1}=P_{k\left(l_{1}-1\right)} a P_{k\left(l_{1}-1\right)}$, where $a \in \mathcal{A}_{k\left(l_{1}-1\right), \mathcal{F}}$ following Proposition 6.2. The proof for Proposition 6.6 and the proof for Proposition 6.8 can be simplified.

Proposition 6.8. Let $A_{i} \in \mathcal{A}_{k(i)}(1 \leq i \leq m)$ be simple products of elements from $S_{i} \subset$ $\mathcal{A}_{k(i), \mathcal{F}} \cup \mathcal{A}_{k(i), \mathcal{B}}$. If the following conditions hold:

(1) There exist $1 \leq l_{1}<l_{2} \leq m$ such that $A_{l_{1}}, A_{l_{1}+1}, \cdots, A_{l_{2}}$ are not Boolean products.

(2) Either $l_{1}=1$ or $A_{l_{1}-1}$ is a Boolean product.

(3) Either $l_{2}=m$ or $A_{l_{2}+1}$ is a Boolean product.

(4) $k(1) \neq k(2) \neq \cdots \neq k(m)$. 
(5) $\mathbb{E}_{\mathcal{L}(\mathcal{X})}\left(A_{l_{1}}\right)=\mathbb{E}_{\mathcal{L}(X)}\left(A_{l_{1}+1}\right)=\cdots=\mathbb{E}_{\mathcal{L}(\mathcal{X})}\left(A_{l_{2}}\right)=0$.

Then, we have $\mathbb{E}_{\mathcal{L}(\mathcal{X})}\left(A_{1} \cdots A_{m}\right)=0$.

Proof. If $1<l_{1}<l_{2}<m$, the asseration follows immediately from Lemma 6.6.

If $l_{2}<m, l_{1}=1$, from the proof of Lemma 6.6. we see that

$$
\begin{aligned}
\left(A_{1} \cdots A_{m}\right) 1_{B} & =\left(A_{l_{1}} \cdots A_{l_{2}}\right)\left(A_{l_{2}+1} \cdots A_{m}\right) 1_{B} \\
& \in\left(\dot{\mathcal{X}}_{k\left(l_{1}\right)} \otimes \cdots \otimes \dot{\mathcal{X}}_{k\left(l_{2}\right)}\right) \oplus\left(\dot{\mathcal{X}}_{k\left(l_{1}\right)} \otimes \cdots \otimes \dot{\mathcal{X}}_{k\left(l_{2}\right)} \otimes \dot{\mathcal{X}}_{k\left(l_{2}+1\right)}\right) .
\end{aligned}
$$

Hence the asseration holds in this case as well.

If $l_{2}=m$, then the assumptions (4) and (5) imply that

$$
\left(A_{l_{1}} \cdots A_{l_{2}}\right) 1_{B} \in\left(\dot{\mathcal{X}}_{k\left(l_{1}\right)} \otimes \cdots \otimes \dot{\mathcal{X}}_{k\left(l_{2}\right)}\right) .
$$

We now have two cases: (i) If further $l_{1}=1$, it is clear that $\mathbb{E}_{\mathcal{L}(\mathcal{X})}\left(A_{1} \cdots A_{m}\right)=0$, thanks to (17). (ii) If $l_{1}>1$, then $A_{l_{1}-1}$ is a Boolean product. We may write $A_{l_{1}-1}=a_{1} b a_{2}$ where $a_{2}$ is a simple product of elements from $\mathcal{A}_{k\left(l_{1}-1\right), \mathcal{F}}$ and $b \in \mathcal{A}_{k\left(l_{1}-1\right), \mathcal{B}}$. If follows that

$$
\begin{aligned}
& a_{2}\left(A_{l_{1}} \cdots A_{m}\right) 1_{B} \in \mathcal{X}_{k\left(l_{1}-1\right)} \otimes\left(\dot{\mathcal{X}}_{k\left(l_{1}\right)} \otimes \cdots \otimes \dot{\mathcal{X}}_{k\left(l_{2}\right)}\right) \\
& \cong\left({\stackrel{\mathcal{X}}{k\left(l_{1}\right)}} \otimes \cdots \otimes \dot{\mathcal{X}}_{k\left(l_{2}\right)}\right) \oplus\left(\dot{\mathcal{X}}_{k\left(l_{1}-1\right)} \otimes \dot{\mathcal{X}}_{k\left(l_{1}\right)} \otimes \cdots \otimes \dot{\mathcal{X}}_{k\left(l_{2}\right)}\right) \text {. }
\end{aligned}
$$

As $b=P_{k\left(l_{1}-1\right)} b P_{k\left(l_{1}-1\right)}$, where $P_{k\left(l_{1}-1\right)}$ is the projection onto $B \oplus \dot{\mathcal{X}}_{k\left(l_{1}-1\right)}$. We have

$$
b a_{2}\left(A_{l_{1}} \cdots A_{m}\right) 1_{B}=0
$$

which implies that $\mathbb{E}_{\mathcal{L}(\mathcal{X})}\left(A_{1} \cdots A_{m}\right)=0$ as well. This finishes the proof.

Proposition-Definition 6.9. Proposition 6.4, Lemma 6.6 and Proposition 6.8 provide us an algorithm for computing mixed moments of free-Boolean independent pairs of random variables and a canonical way to simplify an arbitrary element as follows. Denote by the algebra $\mathcal{A}$ generated by $\bigcup_{i \in \mathcal{I}}\left\{C_{i} \cup D_{i}\right\}$, where $\left\{C_{i}, D_{i}\right\}_{i \in \mathcal{I}}$ is a family of free-Boolean pair of $B$-faces in the $B$-valued probability space $\left(\mathcal{L}(X), \mathbb{E}_{\mathcal{L}(\mathcal{X})}\right)$. That is, $C_{i} \subset \mathcal{A}_{i, \mathcal{F}}, D_{i} \subset \mathcal{A}_{i, \mathcal{B}}$ are subalgebras. Then

$$
\mathcal{A}=\operatorname{span}\left\{\begin{array}{l|l}
A_{1} \cdots A_{m} & \begin{array}{l}
\text { each } A_{i} \text { is a simple product of element from } \mathcal{A}_{\omega(i)}, \\
\text { and } \omega(1) \neq \omega(2) \neq \cdots \neq \omega(m)
\end{array}
\end{array}\right\} .
$$

Let $X=A_{1} \cdots A_{m}$, where each $A_{i}$ is a simple product from $\mathcal{A}_{\omega(i)}$ and $\omega(1) \neq \omega(2) \neq \cdots \neq \omega(m)$. Whenever some $A_{k}(1 \leq k \leq m)$ is a simple product of element from $\mathcal{A}_{\omega(k), \mathcal{F}}$, we replace it by

$$
A_{k}=\left(A_{k}-\mathbb{E}\left(A_{k}\right) 1_{A}\right)+\mathbb{E}\left(A_{k}\right) 1_{A} .
$$

Set $S_{i}=C_{i} \cup D_{i}(i \in \mathcal{I})$. In viewing Lemma 6.6, Proposition 6.2 and the fact that $\mathcal{A}_{i, \mathcal{F}}$ is an algebra, the operator $A_{1} \cdots A_{m}$ can be expressed as the sum of following types of products:

(1) $Z_{(0)}=b 1_{\mathcal{A}}$, where $b \in B$.

(2) $Z_{f}=F_{1} \cdots F_{k}$, where $k \in \mathbb{N}$, each $F_{i} \in C_{\omega(i)} \subset \mathcal{A}_{\omega(i), \mathcal{F}}, \mathbb{E}\left(F_{i}\right)$ and $\omega(1) \neq \cdots \neq \omega(k)$.

(3) $Z_{b}=B_{1} \cdots B_{k}$, where $k \in \mathbb{N}$, each

each $B_{i}$ is a Boolean product of elements from $S_{\omega(i)}$

and $\omega(1) \neq \cdots \neq \omega(k)$. 
(4) $Z_{f b}=F_{1} \cdots F_{k_{1}} B_{1} \cdots B_{k_{2}}$, where $k_{1}, k_{2} \in \mathbb{N}$, each $F_{i} \in C_{\omega(i)} \subset \mathcal{A}_{\omega(i), \mathcal{F}}$ such that $\mathbb{E}\left(F_{i}\right)=$ 0 for $1 \leq i \leq k_{1}$, each $B_{j}$ is a Boolean product of elements from $S_{\omega\left(k_{1}+j\right)}$ for $1 \leq j \leq k_{2}$, and $\omega(1) \neq \cdots \neq \omega\left(k_{1}+k_{2}\right)$.

(5) $Z_{b f}=B_{1} \cdots B_{k_{1}} F_{1} \cdots F_{k_{2}}$, where $k_{1}, k_{2} \in \mathbb{N}$, each $F_{i} \in C_{\omega(i)} \subset \mathcal{A}_{\omega(i), \mathcal{F}}$ such that $\mathbb{E}\left(F_{i}\right)=$ 0 for $1 \leq i \leq k_{2}$, each $B_{j}$ is a Boolean product of elements from $S_{\omega\left(k_{1}+j\right)}$ for $1 \leq j \leq k_{1}$, and $\omega(1) \neq \cdots \neq \omega\left(k_{1}+k_{2}\right)$.

(6) $Z_{f b f}=F_{1} \cdots F_{k_{1}} B_{1} \cdots B_{k_{2}} F_{k_{1}+1} \cdots F_{k_{1}+k_{3}}$, where $k_{1}, k_{2}, k_{3} \in \mathbb{N}$, each $F_{i} \in C_{\omega(i)} \subset$ $\mathcal{A}_{\omega(i), \mathcal{F}}$ for $1 \leq i \leq k_{1}$, each $F_{k_{1}+j} \in C_{\omega\left(k_{1}+k_{2}+j\right)} \subset \mathcal{A}_{\omega\left(k_{1}+k_{2}+j\right), \mathcal{F}}$ for $1 \leq j \leq k_{3}$ such that $\mathbb{E}\left(F_{i}\right)=0$ for $1 \leq i \leq k_{1}+k_{3}, B_{j}$ is a Boolean product of elements from $S_{\omega\left(k_{1}+j\right)}$ for $1 \leq j \leq k_{1}$, and $\omega(1) \neq \cdots \neq \omega\left(k_{1}+k_{2}+k_{3}\right)$.

Furthermore, $\mathbb{E}\left(Z_{f}\right)=0$ by the definition of free independence, $\mathbb{E}\left(Z_{b f}\right)=\mathbb{E}\left(Z_{f b}\right)=\mathbb{E}\left(Z_{f b f}\right)=0$ by Proposition 6.8,

Corollary 6.10. Given an operator $Z_{f b}$ and an operator $Z_{b f}$ of the form in Definition-Proposition 6.9 (4) and (5) respectively, for any $A, B \in \mathcal{A}$, we have

$$
\mathbb{E}\left(Z_{f b} A\right)=\mathbb{E}\left(B Z_{b f}\right)=0 .
$$

Proof. By linearity, it is enough to consider the case when $A$ is the product of simple products. Let $Z_{f b}=F_{1} \cdots F_{k_{1}} B_{1} \cdots B_{k_{2}}$ by definition. The Boolean product $B_{k_{2}}$ is concatenated with some factors in $A$ to be a Boolean product. It then follows from Proposition 6.8 that $\mathbb{E}\left(Z_{f b} A\right)=0$. The other case can be proved in the same way.

Corollary 6.11. Given an operator $Z_{f b f}$ of the form in Definition-Proposition 6.9 (6), for any $A, B \in \mathcal{A}$, we have $\mathbb{E}\left(Z_{f b f} A\right)=\mathbb{E}\left(B Z_{f b f}\right)=0$.

Proof. It is enough to consider the case when $A$ and $B$ are product of simple products. Noticing Proposition 6.6 and applying the simplification method described in Definition-Proposition 6.9, it is easy to see that $Z_{f b f} A$ can be written as the summation of element of the types (3) and (5), whose expectations are zero. Hence $\mathbb{E}\left(Z_{f b f} A\right)=0$. Similary, $B Z_{f b f}$ can be written as the summation of elemtns of the types (4) and (5). Hence $\mathbb{E}\left(B Z_{f b f}\right)=0$.

Lemma 6.12. Let $B_{1} \in \mathcal{A}_{i}$ and $B_{2} \in \mathcal{A}_{j}$ be two Boolean products and $i \neq j$. Then for any $A \in \mathcal{A}$, we have

$$
\mathbb{E}\left(B_{1} B_{2} A\right)=\mathbb{E}\left(B_{1}\right) \mathbb{E}\left(B_{2} A\right) .
$$

Proof. Write $B_{2} A\left(1_{B}\right)=\mathbb{E}\left(B_{2} A\right)+\left(B_{2} A 1_{B}-\mathbb{E}\left(B_{2} A\right)\right) \in B \oplus \dot{\mathcal{X}}_{j}$. We can express the Boolean product $B_{1}$ as $B_{1}=P_{i} a P_{i}$ following Proposition 6.2, and therefore $B_{1}\left(\dot{\mathcal{X}}_{j}\right)=0$, which shows that

$$
\mathbb{E}\left(B_{1} B_{2} A\right)=\mathbb{E}\left(B_{1}\left(\mathbb{E}\left(B_{2} A\right)\right)\right)=\mathbb{E}\left(B_{1}\right) \mathbb{E}\left(B_{2} A\right)
$$

The mixed moments $\mathbb{E}\left(A_{1} \cdots A_{n}\right)$ can be expressed as a universal polynomial of moments of elements in individual algebras $\mathcal{A}_{i}$. Thus we have the following equivalent definition for freeBoolean independence under Moments conditions. 
Proposition 6.13. Let $\left\{\left(C_{i}, D_{i}\right)\right\}_{i \in \mathcal{I}}$ be a family of pairs of algebras in a $B$-valued probability space $(\mathcal{A}, \mathbb{E})$. Set $S_{i}=C_{i} \cup D_{i}$. The family $\left\{\left(C_{i}, D_{i}\right)\right\}_{i \in \mathcal{I}}$ is free-Boolean independent if and only if

(1) whenever $B_{1}, \cdots, B_{m}$ are operators such that:

- for each $1 \leq k \leq m, B_{k}$ is a simple product of elements from $S_{\omega(k)}$, at least one of them is from $D_{\omega(k)}$;

- $\omega(1) \neq \cdots \neq \omega(m)$.

then

$$
\mathbb{E}\left(B_{1} \cdots B_{m}\right)=\mathbb{E}\left(B_{1}\right) \cdots \mathbb{E}\left(B_{m}\right) .
$$

(2) whenever $A_{1}, \cdots, A_{m}$ are operators such that:

- For each $1 \leq k \leq m, A_{k}$ is a product of elements from $S_{\omega(k)}$.

- There exist $1 \leq l_{1}<l_{2} \leq m$ such that $A_{k}$ is a product of elements from $C_{\omega(k)}$ for all $l_{1} \leq k \leq k_{2}$.

- Either $l_{1}=1$ or $A_{l_{1}-1}$ is a product of element from $S_{\omega\left(l_{1}-1\right)}$, at least one of them is in $D_{\omega\left(l_{1}-1\right)}$.

- Either $l_{2}=m$ or $A_{l_{1}+1}$ is a product of element from $S_{\omega\left(l_{1}+1\right)}$, at least one of them is in $D_{\omega\left(l_{2}+1\right)}$.

- $\omega(1) \neq \cdots \neq \omega(m)$.

- $\mathbb{E}\left(A_{l_{1}}\right)=\mathbb{E}\left(A_{l_{1}+1}\right)=\cdots=\mathbb{E}\left(A_{l_{2}}\right)=0$.

Then, we have $\mathbb{E}\left(A_{1} \cdots A_{m}\right)=0$.

\section{Positivity of the amalgamated free-Boolean Product}

In this section, we deal with $B$-functionals with positivity property. For the notion of positivity, we need a $*$-structure on our algebras. We assume the algebra $B$ has a nice positivity structure, i.e. we demand it to be a unital $C^{*}$-algebra. For $*$-algebra $\mathcal{A}$, no such restriction is required.

Definition 7.1. Let $\mathcal{A}$ be a unital $*$-algebra, element $a \in \mathcal{A}$ is said to be positive if there exists a $b \in \mathcal{A}$ such that $a=b b^{*}$. A $B$-linear functional $\mathbb{E}$ is said to be positive if $\mathbb{E}(a)$ is positive for all positive element $a \in \mathcal{A}$. A $B$-linear functional $\mathbb{E}$ is said to be unital if $\mathbb{E}\left(1_{\mathcal{A}}\right)=1_{B}$.

In the rest of this section, we always assume that $\mathcal{A}$ is a unital $*$-algebra and $\mathbb{E}$ is unital. Let $\left\{\left(C_{i}, D_{i}\right)\right\}_{i \in \mathcal{I}}$ ia a family of $B$-faces in a $B$-probability space $(\mathcal{A}, \mathbb{E})$, which generates $\mathcal{A}$. Suppose that the family $\left\{\left(C_{i}, D_{i}\right)\right\}_{i \in \mathcal{I}}$ is free-Boolean independent with amalgamation over $B$ and $C_{i}, D_{i}$ are $*$-subalgebras of $\mathcal{A}$ for all $i \in \mathcal{I}$. For each $i$, let $A_{i}$ be the unital $*$-algebras generated by $C_{i}, D_{i}$. Let $\mathbb{E}_{i}$ be the restriction of $\mathbb{E}$ to $\mathcal{A}_{i}$. Then $\left(\mathcal{A}_{i}, \mathbb{E}_{i}\right)$ is a $B$-valued probability space. We assume that $E_{i}$ is unital and positive and unital for all $i$.

For convenience, we also introduce the following definition. Then, results in PropositionDefinition 6.9 hold in this abstract framework.

Definition 7.2. Given a set $S_{i} \subset C_{i} \cup D_{i}$ and $a_{1}, \cdots, a_{m} \in S_{i}$ their product $A=a_{1} \cdots a_{m}$ is called a simple product of elements from $S_{i}$. It is called a Boolean product of elements from $S_{i}$ if $a_{k} \in S_{i} \cap D_{i}$ for some $1 \leq k \leq m$. 
Recall that in the Section(moments-condition), the algebra $\mathcal{A}$ is the linear span of simple products of type $Z_{0}, Z_{f}, Z_{b}, Z_{b f}, Z_{f s}$ and $Z_{f b f}$, as shown in Proposition-Definition 6.9. Given $Z \in \mathcal{A}$, then $Z$ can be written as

$$
Z=Z_{0}+\sum_{i_{1}} Z_{f}^{\left(i_{1}\right)}+\sum_{i_{2}} Z_{b}^{\left(i_{2}\right)}+\sum_{i_{3}} Z_{b f}^{\left(i_{3}\right)}+\sum_{i_{4}} Z_{f b}^{\left(i_{4}\right)}+\sum_{i_{5}} Z_{f b f}^{\left(i_{5}\right)}
$$

We will show that $\mathbb{E}\left[Z Z^{*}\right]$ is positive.

Remark 7.3. $\left(Z_{b f}^{\left(i_{3}\right)}\right)^{*}$ is a $Z_{f b}$ type element and $\left(Z_{f b}^{\left(i_{4}\right)}\right)^{*}$ is a $Z_{b f}$ type element.

We first note that, by Corollary 6.10 and Corollary 6.11, we have that

$$
\mathbb{E}\left[Z Z^{*}\right]=\mathbb{E}\left[Z_{1} Z_{1}^{*}\right]
$$

where $Z_{1}=Z_{0}+\sum_{i_{1}} Z_{f}^{\left(i_{1}\right)}+\sum_{i_{2}} Z_{b}^{\left(i_{2}\right)}+\sum_{i_{3}} Z_{b f}^{\left(i_{3}\right)}$.

To simplify the notation, we introduce the following notations:

- For operator of the form $Z_{b}=B_{1} \cdots B_{k}$, where $k \in \mathbb{N}$, each $B_{i}$ is a Boolean product of elemnts from $S_{\omega(i)}$ and $\omega(1) \neq \cdots \neq \omega(k)$. Set

$$
\begin{gathered}
\Psi\left(Z_{b}\right)=\mathbb{E}\left(B_{1} \cdots B_{k-1}\right) B_{k}, \quad \text { when } \quad k \geq 2, \\
\Psi^{*}\left(Z_{b}\right) \quad=B_{1} \mathbb{E}\left(B_{2} \cdots B_{k 1}\right), \quad \text { when } \quad k \geq 2,
\end{gathered}
$$

and $\Psi\left(Z_{b}\right)=\Psi^{*}\left(Z_{b}\right)=Z_{b}$ when $k=1$.

- For operators of the form $Z_{b f}=B_{1} \cdots B_{k_{1}} F_{1} \cdots F_{k_{2}}$, where $k_{1}, k_{2} \in \mathbb{N}$, each $F_{i} \in C_{\omega(i)} \subset$ $\mathcal{A}_{\omega(i), \mathcal{F}}$ such that $\mathbb{E}\left(F_{i}\right)=0$ for $1 \leq i \leq k_{2}$, each $B_{j}$ is a Boolean product of elements from $S_{\omega\left(k_{1}+j\right)}$ for $1 \leq j \leq k_{1}$, and $\omega(1) \neq \cdots \neq \omega\left(k_{1}+k_{2}\right)$. Set

$$
\Psi\left(Z_{b f}\right)=\mathbb{E}\left(B_{1} \cdots B_{k_{1}-1}\right)\left(B_{k_{1}} F_{1} \cdots F_{k_{2}}\right), \quad \text { when } \quad k_{1} \geq 2,
$$

and $\Psi\left(Z_{b f}\right)=Z_{b f}=B_{1}\left(F_{1} \cdots F_{k_{2}}\right)$ when $k_{1}=1$.

- For operator of the form $Z_{f b}=F_{1} \cdots F_{k_{1}} B_{1} \cdots B_{k_{2}}$, where $k_{1}, k_{2} \in \mathbb{N}$, each $F_{i} \in C_{\omega(i)} \subset$ $\mathcal{A}_{\omega(i), \mathcal{F}}$ such that $\mathbb{E}\left(F_{i}\right)=0$ for $1 \leq i \leq k_{1}$, each $B_{j}$ is a Boolean product of elemnts from $S_{\omega\left(k_{1}+j\right)}$ for $1 \leq j \leq k_{2}$, and $\omega(1) \neq \cdots \neq \omega\left(k_{1}+k_{2}\right)$. Set

$$
\Psi^{*}\left(Z_{f b}\right)=\left(F_{1} \cdots F_{k_{1}} B_{1}\right) \mathbb{E}\left(B_{2} \cdots B_{k_{2}}\right), \quad \text { when } \quad k_{2} \geq 2,
$$

and $\Psi^{*}\left(Z_{f} b\right)=Z_{f b}$ when $k_{2}=1$.

Note that $Z_{b f}^{*}$ is of the same type as $Z_{f b}$ following Proposition-Defintion 6.9 (4), (5). It is easy to check that the following relation holds:

$$
\Psi\left(Z_{b f}\right)^{*}=\Psi^{*}\left(Z_{b f}^{*}\right) .
$$

Lemma 7.4. Let $Z_{b f}=B_{1} \cdots B_{k_{1}} F_{1} \cdots F_{k_{2}}$, where each $F_{i} \in \mathcal{A}_{\omega(i), \mathcal{F}}$ such that $\mathbb{E}\left(F_{i}\right)=0$ for $1 \leq i \leq k_{2}$, each $B_{j}$ is a Boolean product from $\mathcal{A}_{\omega\left(k_{1}+j\right)}$ for $1 \leq j \leq k_{1}$, and $\omega(1) \neq \cdots \neq$ $\omega\left(k_{1}+k_{2}\right)$. Then, $\mathbb{E}\left[Z_{b f} Z^{\prime}\right]=\mathbb{E}\left[\Psi\left(Z_{b f}\right) Z^{\prime}\right]$ for all simple products $Z^{\prime}$.

Proof. It follows by applying Lemma 6.12 inductively. 
Lemma 7.5. Let $Z_{f b}=F_{1} \cdots F_{k_{1}} B_{1} \cdots B_{k_{2}}$, where each $F_{i} \in \mathcal{A}_{\omega(i), \mathcal{F}}$ such that $\mathbb{E}\left(F_{i}\right)=0$ for $1 \leq i \leq k_{1}, k_{1} \geq 0, k_{2} \geq 1$ each $B_{j}$ is a Boolean product from $\mathcal{A}_{\omega\left(k_{1}+j\right)}$ for $k_{1}<j \leq k_{1}+k_{2}$, and $\omega(1) \leq \omega\left(k_{1}+k_{2}\right)$. Then, $\mathbb{E}\left[Z^{\prime} Z_{f b}\right]=\mathbb{E}\left[Z^{\prime} \Psi^{*}\left(Z_{f b}\right)\right]$ for all simple products $Z^{\prime}$.

Proof. It follows by applying Lemma 6.3 inductively.

Lemma 7.6. $\mathbb{E}\left[Z_{1} Z_{1}^{*}\right]=\mathbb{E}\left[Z_{2} Z_{2}^{*}\right]$, where $Z_{2}=Z_{0}+\sum_{i_{1}} Z_{f}^{\left(i_{1}\right)}+\Psi\left(\sum_{i_{2}} Z_{b}^{\left(i_{2}\right)}+\sum_{i_{3}} Z_{b f}^{\left(i_{3}\right)}\right)$.

Proof. Appy Lemma 7.4 and Lemma 7.5, we have

$$
\begin{aligned}
& \mathbb{E}\left[Z_{1} Z_{1}^{*}\right] \\
= & \mathbb{E}\left[\left(Z_{0}+\sum_{i_{1}} Z_{f}^{\left(i_{1}\right)}+\sum_{i_{2}} Z_{b}^{\left(i_{2}\right)}+\sum_{i_{3}} Z_{b f}^{\left(i_{3}\right)}\right)\left(Z_{0}^{*}+\sum_{i_{1}} Z_{f}^{\left(i_{1}\right)}+\sum_{i_{2}} Z_{b}^{\left(i_{2}\right)}+\sum_{i_{3}} Z_{b f}^{\left(i_{3}\right)}\right)^{*}\right] \\
= & \mathbb{E}\left[\left(Z_{0}+\sum_{i_{1}} Z_{f}^{\left(i_{1}\right)}+\sum_{i_{2}} Z_{b}^{\left(i_{2}\right)}+\sum_{i_{3}} Z_{b f}^{\left(i_{3}\right)}\right)\left(Z_{0}^{*}+\sum_{i_{1}} Z_{f}^{\left(i_{1}\right) *}+\sum_{i_{2}} Z_{b}^{\left(i_{2}\right) *}+\sum_{i_{3}} Z_{b f}^{\left(i_{3}\right) *}\right)\right] \\
= & \mathbb{E}\left[\left(Z_{0}+\sum_{i_{1}} Z_{f}^{\left(i_{1}\right)}+\Psi\left(\sum_{i_{2}} Z_{b}^{\left(i_{2}\right)}+\sum_{i_{3}} Z_{b f}^{\left(i_{3}\right)}\right)\right)\left(Z_{0}^{*}+\sum_{i_{1}} Z_{f}^{\left(i_{1}\right) *}+\left(\sum_{i_{2}} Z_{b}^{\left(i_{2}\right) *}+\sum_{i_{3}} Z_{b f}^{\left(i_{3}\right) *}\right)\right)\right] \\
= & \mathbb{E}\left[\left(Z_{0}+\sum_{i_{1}} Z_{f}^{\left(i_{1}\right)}+\Psi\left(\sum_{i_{2}} Z_{b}^{\left(i_{2}\right)}+\sum_{i_{3}} Z_{b f}^{\left(i_{3}\right)}\right)\right)\left(Z_{0}^{*}+\sum_{i_{1}} Z_{f}^{\left(i_{1}\right) *}+\Psi^{*}\left(\sum_{i_{2}} Z_{b}^{\left(i_{2}\right) *}+\sum_{i_{3}} Z_{b f}^{\left(i_{3}\right) *}\right)\right)\right] \\
= & \mathbb{E}\left[\left(Z_{0}+\sum_{i_{1}} Z_{f}^{\left(i_{1}\right)}+\Psi\left(\sum_{i_{2}} Z_{b}^{\left(i_{2}\right)}+\sum_{i_{3}} Z_{b f}^{\left(i_{3}\right)}\right)\right)\left(Z_{0}^{*}+\sum_{i_{1}} Z_{f}^{\left(i_{1}\right) *}+\left(\Psi^{*}\left(\sum_{i_{2}} Z_{b}^{\left(i_{2}\right)}+\sum_{i_{3}} Z_{b f}^{\left(i_{3}\right)}\right)\right)^{*}\right)\right] \\
= & \mathbb{E}\left[Z_{2} Z_{2}^{*}\right]
\end{aligned}
$$

Notice that $\Psi\left(Z_{b}^{\left(i_{2}\right)}\right)$ is a simple Boolean product and $\Psi\left(Z_{b f}^{\left(i_{3}\right)}\right)$ can be written as $B_{i_{3}} Z_{f^{\prime}}^{\left(i_{3}\right)}$ where $Z_{f^{\prime}}^{\left(i_{3}\right)}$ is the type (3) of product in Proposition-Definition [6.9. According to the length of the word appearing in the expression of $Z_{2}$ defined in Lemma 7.6, we can then rewrite $Z_{2}$ as

$$
Z_{2}=b 1_{\mathcal{A}}+\sum_{k=1}^{n} \sum_{r=1}^{m_{k}} a_{k, r, 1} a_{k, r, 2} \cdots a_{k, r, k}
$$

where $a_{k, r, p} \in D_{\omega_{k, r}(p)}$ (the right face) for $p \geq 2$, and $a_{k, r, 1} \in S_{\omega_{k, r}(p)}$ (either left face or right face), $\mathbb{E}\left(a_{k, r, p}\right)=0$ and $\omega_{k, r}:\{1, \cdots, k\} \rightarrow \mathcal{I}$ such that $\omega_{k, r}(1) \neq \cdots \neq \omega_{k, r}(k)$.

We now have the following result.

Lemma 7.7. Let $a_{1} \in \mathcal{A}_{\omega_{1}(1)}, a_{i} \in \mathcal{A}_{\omega_{1}(i), \mathcal{F}}$ for $i=2, \ldots, n$ such that $\mathbb{E}\left(a_{i}\right)=\mathbb{E}\left(\widetilde{a}_{i}\right)=0$ and $\omega_{1}:\{1, \cdots, n\} \rightarrow \mathcal{I}, \omega_{1}(1) \neq \cdots \neq \omega_{1}(n)$. Let $\widetilde{a}_{1} \in \mathcal{A}_{\omega_{2}(1)}, \widetilde{a}_{j} \in \mathcal{A}_{\omega_{2}(j), \mathcal{F}}$ for $i=2, \ldots, m$ such that $\omega_{2}:\{1, \cdots, m\} \rightarrow \mathcal{I}, \omega_{2}(1) \neq \cdots \neq \omega_{1}(m)$. We then have

$$
\mathbb{E}\left[a_{1} a_{2} \cdots a_{n-1} a_{n} \widetilde{a}_{m} \widetilde{a}_{m-1} \cdots \widetilde{a}_{1}\right]=\delta_{n, m} \mathbb{E}\left[a_{1} a_{2} \cdots \mathbb{E}\left[a_{n-1} \mathbb{E}\left[a_{n} \widetilde{a}_{m}\right] \widetilde{a}_{m-1}\right] \cdots \widetilde{a}_{1}\right] .
$$

Proof. When $n=0$ or $m=0$, there is nothing to prove. It is sufficient to prove that

$$
\mathbb{E}\left[a_{1} a_{2} \cdots a_{n-1} a_{n} \widetilde{a}_{m} \widetilde{a}_{m-1} \cdots \widetilde{a}_{1}\right]=\delta_{n, m} \mathbb{E}\left[a_{1} a_{2} \cdots a_{n-1} \mathbb{E}\left[a_{n} \widetilde{a}_{m}\right] \widetilde{a}_{m-1} \cdots \widetilde{a}_{1}\right] .
$$

Notice that if $\omega_{1}(n) \neq \omega_{2}(m)$, then by Proposition 6.8 and the definition of freeness, then, by Proposition 6.8, we have

$$
\mathbb{E}\left[a_{1} a_{2} \cdots a_{n-1} a_{n} \widetilde{a}_{m} \widetilde{a}_{m-1} \cdots \widetilde{a_{1}}\right]=\mathbb{E}\left[a_{n} \widetilde{a}_{m}\right]=0 .
$$


On the other hand, if $\omega_{1}(n)=\omega_{2}(m)$, notice that $a_{n} \widetilde{a}_{m}-\mathbb{E}\left[a_{n} \widetilde{a}_{m}\right] \in \mathcal{A}_{\omega_{1}(n)}, \omega_{1}(n) \neq \omega_{1}(n-1)$ and $\omega_{1}(n) \neq \omega_{2}(m-1)$, then we have

$$
\mathbb{E}\left[a_{1} a_{2} \cdots a_{n-1}\left(a_{n} \widetilde{a}_{m}-\mathbb{E}\left[a_{n} \widetilde{a}_{m}\right]\right) \widetilde{a}_{m-1} \cdots \widetilde{a}_{1}\right]=0,
$$

which is the desired equation.

Therefore, we have the following equation.

$$
\mathbb{E}\left[Z_{2} Z_{2}^{*}\right]=b b^{*}+\sum_{k=1}^{n} \mathbb{E}\left[\left(\sum_{r=1}^{m_{k}} a_{k, r, 1} a_{k, r, 2} \cdots a_{k, r^{\prime}, k}\right)\left(\sum_{r^{\prime}=1}^{m_{k}} a_{k, r^{\prime}, 1} a_{k, r^{\prime}, 2} \cdots a_{k, r^{\prime}, k}\right)^{*}\right] .
$$

Moreover, Lemma 7.7 implies that the term $\mathbb{E}\left[\left(a_{k, r, 1} a_{k, r, 2} \cdots a_{k, r^{\prime}, k}\right)\left(a_{k, r^{\prime}, 1} a_{k, r^{\prime}, 2} \cdots a_{k, r^{\prime}, k}\right)^{*}\right]$ is not vanishing only if $\omega_{k, r}=\omega_{k, r^{\prime}}$. Let $\sim$ be the equivalence relation on $\left\{1, \cdots, m_{k}\right\}$ such that $l_{1} \sim l_{2}$ if and only if $\omega_{k, l_{1}, k}=\omega_{k, l_{2}, k}$. Let $\left\{V_{1}, \cdots, V_{s}\right\}$ be the family of equivalence classes of $\left\{1, \cdots, m_{k}\right\}$. Then

$$
\begin{aligned}
& \mathbb{E}\left[\left(\sum_{r=1}^{m_{k}} a_{k, r, 1} a_{k, r, 2} \cdots a_{k, r^{\prime}, k}\right)\left(\sum_{r^{\prime}=1}^{m_{k}} a_{k, r^{\prime}, 1} a_{k, r^{\prime}, 2} \cdots a_{k, r^{\prime}, k}\right)^{*}\right] \\
= & \mathbb{E}\left[\left(\sum_{r \in V_{l}} a_{k, r, 1} a_{k, r, 2} \cdots a_{k, r^{\prime}, k}\right)\left(\sum_{r^{\prime} \in V_{l}} a_{k, r^{\prime}, 1} a_{k, r^{\prime}, 2} \cdots a_{k, r^{\prime}, k}\right)^{*}\right]
\end{aligned}
$$

To show that $E\left[Z_{2} Z_{2}^{*}\right]$ is positive, we just need to show that

$$
\mathbb{E}\left[\left(\sum_{r \in V_{l}} a_{k, r, 1} a_{k, r, 2} \cdots a_{k, r^{\prime}, k}\right)\left(\sum_{r^{\prime} \in V_{l}} a_{k, r^{\prime}, 1} a_{k, r^{\prime}, 2} \cdots a_{k, r^{\prime}, k}\right)^{*}\right] \geq 0
$$

for all $k$.

This is exactly the circumstance in the proof of [16, Proposition 3.5.6]. Therefore, we have reduced the positivity question of $\mathbb{E}\left[Z Z^{*}\right]$ to a known result in free probability context. Since $Z$ is arbitrary, we thus have the following theorem.

Theorem 7.8. Let $\left\{\left(C_{i}, D_{i}\right)\right\}_{i \in \mathcal{I}}$ be a family of $B$-faces in a $B$-probability space $(\mathcal{A}, \mathbb{E})$, which generates $\mathcal{A}$. We assume that $C_{i}, D_{i}$ are $*$-subalgebras of $\mathcal{A}$ for all $\mathcal{A}$. For each $i$, let $\mathcal{A}_{i}$ be the *-algebras generated by $C_{i}, D_{i}$. We assume that the restriction of $\mathbb{E}$ to $\mathcal{A}_{i}$ is positive. If the family $\left\{\left(C_{i}, D_{i}\right)\right\}_{i \in \mathcal{I}}$ is free-Boolean independent with amalgamation over $B$, then $\mathbb{E}$ is positive.

Acknowledgement The second-named author would like to thank Professor Hari Bercovici for the invitation to visit Indiana University, where the authors can meet and part of this work was done. He also wants to thank Professor Alexandru Nica for his continued support. This project was partially supported by NSFC No. 11501423, 11431011. 


\section{ReFERENCES}

[1] Marek Bożejko, Michael Leinert, and Roland Speicher. Convolution and limit theorems for conditionally free random variables. Pacific J. Math., 175(2):357-388, 1996.

[2] Ian Charlesworth, Brent Nelson, and Paul Skoufranis. Combinatorics of bi-freeness with amalgamation. Comm. Math. Phys., 338(2):801-847, 2015.

[3] Yinzheng Gu, Takahiro Hasebe, and Paul Skoufanis. Bi-monotonic independence for pairs of algebras. arXiv:1708.05334, 2017.

[4] Yinzheng Gu and Paul Skoufanis. Bi-boolean independence for pairs of algebras. arXiv:1703.03072, to appear in Complex Anal. Oper. Theory, 2017.

[5] Yinzheng Gu and Paul Skoufranis. Conditional bi-free independence with amalgamation,. arXiv:1609.07820, to appear in Int. Math. Res. Not. IMRN, 2017.

[6] Yinzheng Gu and Paul Skoufranis. Conditionally bi-free independence for pairs of faces. J. Funct. Anal., 273(5):1663-1733, 2017.

[7] Weihua Liu. A noncommutative de Finetti theorem for boolean independence. J. Funct. Anal., 269(7):19501994, 2015.

[8] Weihua Liu. Free-boolean independence for pairs of algebras. arXiv:1710.01374, 2017.

[9] Wojciech Mł otkowski. Operator-valued version of conditionally free product. Studia Math., 153(1):13-30, 2002.

[10] Naofumi Muraki. The five independences as natural products. Infin. Dimens. Anal. Quantum Probab. Relat. Top., 6(3):337-371, 2003.

[11] Alexandru Nica and Roland Speicher. Lectures on the combinatorics of free probability, volume 335 of London Mathematical Society Lecture Note Series. Cambridge University Press, Cambridge, 2006.

[12] Mihai Popa. A combinatorial approach to monotonic independence over a $C^{*}$-algebra. Pacific J. Math., 237(2):299-325, 2008.

[13] Mihai Popa. A new proof for the multiplicative property of the Boolean cumulants with applications to the operator-valued case. Colloq. Math., 117(1):81-93, 2009.

[14] Gian-Carlo Rota. On the foundations of combinatorial theory. I. Theory of Möbius functions. Z. Wahrscheinlichkeitstheorie und Verw. Gebiete, 2:340-368 (1964), 1964.

[15] Roland Speicher. On universal products. In Free probability theory (Waterloo, ON, 1995), volume 12 of Fields Inst. Commun., pages 257-266. Amer. Math. Soc., Providence, RI, 1997.

[16] Roland Speicher. Combinatorial theory of the free product with amalgamation and operator-valued free probability theory. Mem. Amer. Math. Soc., 132(627):x+88, 1998.

[17] Roland Speicher and Reza Woroudi. Boolean convolution. In Free probability theory (Waterloo, ON, 1995), volume 12 of Fields Inst. Commun., pages 267-279. Amer. Math. Soc., Providence, RI, 1997.

[18] Dan Voiculescu. Operations on certain non-commutative operator-valued random variables. Astérisque, (232):243-275, 1995. Recent advances in operator algebras (Orléans, 1992).

[19] Dan-Virgil Voiculescu. Free probability for pairs of faces I. Comm. Math. Phys., 332(3):955-980, 2014.

Weihua Liu: Department of Mathematics, Indiana University, Boomington, IN 47401, USA., E-mail address: liuweih@indiana.edu

Ping Zhong: Department of Pure Mathematics, University of Waterloo, 200 University Avenue West, Waterloo, ON, N2L 3G1, Canada.

E-mail address: ping.zhong@uwaterloo.ca 